\title{
RELICS: Reionization Lensing Cluster Survey
}

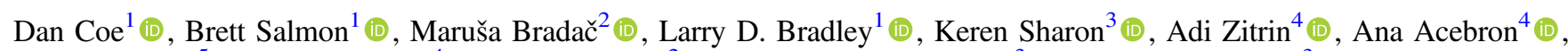
Catherine Cerny ${ }^{5}$, Nathália Cibirka ${ }^{4}$ (1), Victoria Strait ${ }^{2}$ (1) , Rachel Paterno-Mahler $^{3}$ (10), Guillaume Mahler ${ }^{3}$ (1), Roberto J. Avila ${ }^{1}$, Sara Ogaz ${ }^{1}$, Kuang-Han Huang ${ }^{2}$ (1) , Debora Pelliccia ${ }^{2,6}$, Daniel P. Stark ${ }^{7}$, Ramesh Mainali ${ }^{7}$ (1), Pascal A. Oesch ${ }^{8,9}$ (1), Michele Trenti ${ }^{10,11}$ (1) , Daniela Carrasco ${ }^{10}$ (1) , William A. Dawson $^{12}{ }^{(10}$, Steven A. Rodney ${ }^{13}$ (1) , Louis-Gregory Strolger ${ }^{1}$, Adam G. Riess ${ }^{1}$, Christine Jones ${ }^{14}$, Brenda L. Frye ${ }^{7}$, Nicole G. Czakon ${ }^{15}$, Keiichi Umetsu ${ }^{15}$ (1) , Benedetta Vulcani ${ }^{16}$ (1), Or Graur $^{14,17,35}$ (1), Saurabh W. Jha ${ }^{18}$ (1), Melissa L. Graham ${ }^{19}$ (1), Alberto Molino ${ }^{20,21}$, Mario Nonino ${ }^{22}$, Jens Hjorth ${ }^{23}$ (1), Jonatan Selsing ${ }^{9,24}$ (1) , Lise Christensen ${ }^{23}$ (1) , Shotaro Kikuchihara ${ }^{25,26}$ (1) , Masami Ouchi $^{25,27}$ (1), Masamune Oguri ${ }^{27,28,29}$ (1)

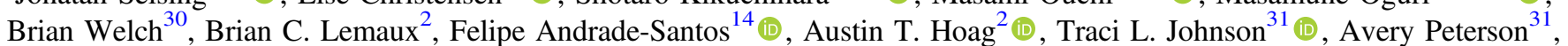
Matthew Past ${ }^{31}$, Carter Fox ${ }^{3}$, Irene Agulli ${ }^{4}$, Rachael Livermore ${ }^{10,11}$ (D), Russell E. Ryan ${ }^{1}$, Daniel Lam ${ }^{32}$ (D), Irene Sendra-Server ${ }^{33}$, Sune Toft ${ }^{9,24}$ (1) , Lorenzo Lovisari ${ }^{14}$, and Yuanyuan $\mathrm{Su}^{34}$

${ }^{1}$ Space Telescope Science Institute, 3700 San Martin Drive, Baltimore, MD 21218, USA; DCoe@STScI.edu ${ }^{2}$ Department of Physics, University of California, Davis, CA 95616, USA

${ }^{3}$ Department of Astronomy, University of Michigan, 1085 South University Avenue, Ann Arbor, MI 48109, USA

${ }^{4}$ Physics Department, Ben-Gurion University of the Negev, P.O. Box 653, Beer-Sheva 84105, Israel

${ }^{5}$ Astronomy Department and Institute for Astrophysical Research, Boston University, 725 Commonwealth Avenue, Boston, MA 02215, USA ${ }^{6}$ Department of Physics and Astronomy, University of California, Riverside, CA 92521, USA

${ }^{7}$ Department of Astronomy, Steward Observatory, University of Arizona, 933 North Cherry Avenue, Tucson, AZ 85721, USA

${ }^{8}$ Department of Astronomy, University of Geneva, Chemin des Maillettes 51, 1290 Versoix, Switzerland

${ }^{9}$ Cosmic Dawn Center (DAWN), Copenhagen, Denmark

${ }^{10}$ School of Physics, University of Melbourne, VIC 3010, Australia

${ }^{11}$ ARC Centre of Excellence for All Sky Astrophysics in 3 Dimensions (ASTRO 3D), VIC 2010, Australia

${ }^{12}$ Lawrence Livermore National Laboratory, P.O. Box 808 L-210, Livermore, CA 94551, USA

${ }^{13}$ Department of Physics and Astronomy, University of South Carolina, 712 Main Street, Columbia, SC 29208, USA

${ }^{14}$ Harvard-Smithsonian Center for Astrophysics, 60 Garden Street, Cambridge, MA 02138, USA

${ }_{15}$ Academia Sinica Institute of Astronomy and Astrophysics (ASIAA), No. 1, Section 4, Roosevelt Road, Taipei 10617, Taiwan

${ }^{16}$ INAF-Osservatorio Astronomico di Padova, Vicolo Dell'osservatorio 5, I-35122 Padova, Italy

${ }_{17}^{17}$ Department of Astrophysics, American Museum of Natural History, Central Park West and 79th Street, New York, NY 10024-5192, USA

${ }^{18}$ Department of Physics and Astronomy, Rutgers, The State University of New Jersey, 136 Frelinghuysen Road, Piscataway, NJ 08854, USA

${ }^{19}$ Department of Astronomy, University of Washington, Box 351580, U.W., Seattle, WA 98195-1580, USA

${ }^{20}$ Universidade de São Paulo, Instituto de Astronomia, Geofísica e Ciências Atmosféricas, Rua do Matão 1226, São Paulo, Brazil ${ }^{21}$ Instituto de Astrofísica de Andalucía (IAA-CSIC), E-18080 Granada, Spain

${ }^{22}$ INAF-Osservatorio Astronomico di Trieste, Via Tiepolo 11, I-34131 Trieste, Italy

${ }^{23}$ DARK, Niels Bohr Institute, University of Copenhagen, Lyngbyvej 2, DK-2100 Copenhagen, Denmark

${ }^{24}$ Niels Bohr Institute, University of Copenhagen, Lyngbyvej 2, DK-2100, Copenhagen, Denmark

${ }^{25}$ Institute for Cosmic Ray Research, The University of Tokyo, 5-1-5 Kashiwanoha, Kashiwa, Chiba 277-8582, Japan

${ }^{26}$ Department of Astronomy, Graduate School of Science, The University of Tokyo, 7-3-1 Hongo, Bunkyo, Tokyo, 113-0033, Japan

${ }^{27}$ Kavli Institute for the Physics and Mathematics of the Universe (Kavli IPMU, WPI), University of Tokyo, Kashiwa, Chiba 277-8583, Japan

${ }^{28}$ Research Center for the Early Universe, The University of Tokyo, 7-3-1 Hongo, Bunkyo-ku, Tokyo 113-0033, Japan

${ }^{29}$ Department of Physics, The University of Tokyo, 7-3-1 Hongo, Bunkyo-ku, Tokyo 113-0033, Japan

${ }^{30}$ Department of Physics and Astronomy, Johns Hopkins University, 3400 North Charles Street, Baltimore, MD 21218, USA

${ }^{31}$ Department of Astronomy, University of Michigan, 1085 South University Drive, Ann Arbor, MI 48109, USA

${ }^{32}$ Leiden Observatory, Leiden University, NL-2300 RA Leiden, The Netherlands

${ }^{33}$ Department of Theoretical Physics, University of the Basque Country UPV/EHU, E-48080 Bilbao, Spain

${ }^{34}$ Department of Physics and Astronomy, University of Kentucky, 505 Rose Street, Lexington, KY 40506, USA

Received 2019 March 5; revised 2019 August 6; accepted 2019 September 2; published 2019 October 14

\begin{abstract}
Large surveys of galaxy clusters with the Hubble Space Telescope (HST) and Spitzer, including the Cluster Lensing And Supernova survey with Hubble and the Frontier Fields, have demonstrated the power of strong gravitational lensing to efficiently deliver large samples of high-redshift galaxies. We extend this strategy through a wider, shallower survey named RELICS, the Reionization Lensing Cluster Survey, described here. Our 188-orbit Hubble Treasury Program observed 41 clusters at $0.182 \leqslant z \leqslant 0.972$ with Advanced Camera for Surveys (ACS) and WFC3/IR imaging spanning 0.4-1.7 $\mu \mathrm{m}$. We selected 21 of the most massive clusters known based on Planck PSZ2 estimates and 20 additional clusters based on observed or inferred lensing strength. RELICS observed 46 WFC3/IR pointings ( $\left.200 \mathrm{arcmin}^{2}\right)$ each with two orbits divided among four filters (F105W, F125W, F140W, and F160W) and ACS imaging as needed to achieve single-orbit depth in each of three filters (F435W, F606W, and F814W). As previously reported by Salmon et al., we discovered over $300 z \sim 6-10$ candidates, including the brightest $z \sim 6$ candidates known, and the most distant spatially resolved lensed arc known at $z \sim 10$. Spitzer IRAC imaging (945 hr awarded, plus 100 archival, spanning 3.0-5.0 $\mu \mathrm{m}$ ) has crucially enabled us to distinguish $z \sim 10$ candidates from $z \sim 2$ interlopers. For each cluster, two HST observing epochs were staggered by about a month, enabling us to discover 11 supernovae, including 3 lensed supernovae, which we followed up with
\end{abstract}

\footnotetext{
35 NSF Astronomy and Astrophysics Postdoctoral Fellow.
} 
20 orbits from our program. Reduced $H S T$ images, catalogs, and lens models are available on MAST, and reduced Spitzer images are available on IRSA.

Key words: dark ages, reionization, first stars - dark matter - galaxies: clusters: general - galaxies: high-redshift gravitational lensing: strong - supernovae: general

\section{Introduction}

Gravitational lensing magnification by massive galaxy clusters has a long history of helping astronomers discover the most distant galaxies known with the Hubble Space Telescope (HST) and Spitzer (see Kneib \& Natarajan 2011 Section 5.8 for a review). Twenty years ago, a $z=4.92$ galaxy lensed by the cluster MS $1358+62$ was the most distant known (Franx et al. 1997). Ten years ago, that record belonged to A1689-zD1 at $z \sim 7.5$ (Bradley et al. 2008; Watson et al. 2015). More recently, the Cluster Lensing And Supernova survey with Hubble (CLASH; Postman et al. 2012) yielded the triply imaged $z \sim 10.8$ candidate MACS0647-JD (Coe et al. 2013; Pirzkal et al. 2015; Chan et al. 2017). This redshift has been surpassed only by the grism measurement of $z=11.1$ (Oesch et al. 2016) for GN-z11 discovered by Oesch et al. 2014 in blank-field imaging by the CANDELS program (Grogin et al. 2011; Koekemoer et al. 2011). Both $z \sim 11$ galaxies are similarly bright at $1.6 \mu \mathrm{m}$ (F160W AB mag 25.9), making them excellent targets for follow-up study with the James Webb Space Telescope (JWST).

For a given observing strategy, lensed fields generally yield significantly more high-redshift galaxies than blank fields, especially at the highest redshifts and in relatively shallow imaging (e.g., Coe et al. 2015). Lensing does sacrifice highredshift search volume owing to the magnification and the foreground cluster light. But lensing more than compensates for the lost volume by magnifying many more faint galaxies into view whenever luminosity function number counts are steeper than $\phi \propto L^{-2}$ (Broadhurst et al. 1995). This is especially true brightward of $L^{*}$, for example, $\mathrm{AB}$ mag $\sim 28$ at $z \sim 8$ (Bradley et al. 2012; Finkelstein 2016).

CLASH demonstrated this lensing advantage, yielding significantly more galaxies at $z \sim 6-8$ than blank-field surveys (Bradley et al. 2014). CLASH obtained 20-orbit HST imaging for each of 25 clusters in 16 filters, including F160W observed to AB mag 27.5 ( $5 \sigma$ depth; Postman et al. 2012; Molino et al. 2017). The first 18 clusters yielded 262 candidates at $z \sim 6-8$ (Bradley et al. 2014), plus a few at $z \sim 9-11$ (Zheng et al. 2012; Coe et al. 2013; Bouwens et al. 2014).

The Frontier Fields (Lotz et al. 2017) obtained deeper 140-orbit $H S T$ imaging for each of six clusters and six blank parallel fields in seven filters, including F160W observed to AB mag 28.7. At these depths, $\phi \propto L^{-2}$ for high-redshift galaxies (the luminosity function faint-end slope $\alpha \sim-2$ roughly at $z \sim 6-8$ ), so the lensed and blank-field high- $z$ counts were predicted to be similar (Coe et al. 2015). Indeed, the Frontier Fields yielded similar total numbers of lensed and blank-field high-redshift galaxies, altogether 453 at $z \sim 6-9$ from one analysis (Kawamata et al. 2018), plus a few $z \sim 10$ candidates (Zitrin et al. 2014; Infante et al. 2015; McLeod et al. 2016; Oesch et al. 2018). Due to their magnifications, the lensed galaxies are intrinsically fainter than the blank-field galaxies. By combining deep HST imaging with the power of gravitational lensing, the Frontier Fields revealed the faintest galaxies yet known, including some of those likely responsible for reionization at $z \sim 6$ (Livermore et al. 2017; Atek et al. 2018).
While CLASH and the Frontier Fields yielded many highredshift candidates, neither survey was optimized to deliver high-redshift candidates observed brightly enough for detailed follow-up study with current and future observatories. Detailed studies are required to determine galaxies' ages, stellar masses, compositions, and ionizing strengths (see Stark 2016 for a review). This knowledge can further inform how early galaxies enriched and reionized the universe (see Dayal \& Ferrara 2018 for a review). It is imperative to use existing facilities to discover the best targets for study, ideally before JWST Cycle 1. Gravitational lensing offers the most efficient route to do this. The Planck all-sky survey delivered the PSZ2 catalog of $>1000$ massive galaxy clusters (Planck Collaboration et al. 2016a) detected via their Sunyaev-Zel'dovich (SZ) effect (Sunyaev \& Zeldovich 1970) on the cosmic microwave background (CMB). By searching the PSZ2 catalog, we found that many massive clusters (including presumably excellent lenses) lacked the combination of HST Advanced Camera for Surveys (ACS) and Wide Field Camera 3 infrared (WFC3/IR) imaging required to discover high-redshift candidates at $z>6$.

RELICS embarked on an efficient survey with $H S T$ and Spitzer to discover the best and brightest high-redshift galaxies (Coe 2018). RELICS obtained five-orbit HST imaging of 41 clusters in seven filters (the same filters used by the Frontier Fields), including F160W to AB mag 26.5. Our relatively shallow imaging covered more area than CLASH or the Frontier Fields, yielding high-redshift candidates that are brighter, either intrinsically and/or due to lensing magnification. See Table 1 for a summary comparison of CLASH, the Frontier Fields, and RELICS. More recently, a new HST program, BUFFALO (PIs Steinhardt \& Jauzac; GO 15117), has begun, extending the Frontier Fields to the wider area covered by deep Spitzer imaging.

Also notable and inspiring for this project are the HST Snapshot programs observing galaxy clusters discovered by the MAssive Cluster Survey (MACS; Ebeling et al. 2001). Shallower HST imaging ( $\sim 1$ orbit per cluster) has been obtained for 86 clusters to date (Repp \& Ebeling 2018). For 29 of these clusters, four-band imaging, including F140W to AB mag 26.6, was completed, yielding 20 candidates at $z \sim 7-9$ (Repp et al. 2016). Nine MACS clusters are included in RELICS. Prior to RELICS, they had archival HST ACS and/or WFPC2 imaging, but not WFC3/IR or NICMOS.

Meanwhile, large Spitzer programs such as SURFS UP (Bradač et al. 2014; Ryan et al. 2014; Huang et al. 2016) and the Frontier Fields have also surveyed many galaxy clusters, helping to identify high-redshift galaxies and study their properties (e.g., Hoag et al. 2019). The Grism Lens-Amplified Survey from Space (Treu et al. 2015) delivered spectroscopic properties and high-redshift confirmations (e.g., Schmidt et al. 2016, 2017). The high-redshift searches in lensing programs complement searches in blank-field surveys such as the UDF, CANDELS, and BoRG, constraining luminosity functions from $z \sim 4$ to 10 (Bouwens et al. 2015; Finkelstein 2016; Morishita et al. 2018; Oesch et al. 2018). 
Table 1

Recent Large HST Cluster Lensing Surveys

\begin{tabular}{lccc}
\hline \hline & CLASH & Frontier Fields & RELICS \\
\hline Clusters & 25 & 6 & 41 \\
$H S T$ orbits per cluster & $20^{\mathrm{a}}$ & 140 & $5^{\mathrm{a}}$ \\
Total $H S T$ orbits & 524 & 840 & 188 \\
Supernova orbits & 50 & 0 & 20 \\
$H S T$ filters & 16 & 7 & 7 \\
Depth in F160W & 27.5 & 28.7 & 26.5 \\
$H S T$ Cycle numbers & $18-20$ & $21-23$ & 23 \\
$H S T$ begin & Nov 10 & Oct 13 & Oct 15 \\
$H S T$ end & Jul 13 & Sept 16 & Apr 17 \\
\hline
\end{tabular}

Notes.

a Depth including archival HST imaging.

b HST orbits allocated specifically for supernova follow-up.

${ }^{\mathrm{c}} \mathrm{AB}$ mag $5 \sigma$ depth for point sources.

Below we discuss the RELICS science drivers (Section 2); galaxy cluster targets (Section 3); observations with HST, Spitzer, and other observatories (Section 4); the image reductions and catalogs (Section 5); results to date (Section 6); and a summary (Section 7).

We use the $\mathrm{AB}$ magnitude system, $m_{\mathrm{AB}}=31.4-2.5 \log$ $\left(f_{\nu} /\right.$ nJy) (Oke 1974; Oke \& Gunn 1983). Where needed, we assume a flat concordance $\Lambda$ CDM cosmology with $h=0.7$, $\Omega_{m}=0.3$, and $\Omega_{\Lambda}=0.7$, where $H_{0}=100 h \mathrm{~km} \mathrm{~s}^{-1} \mathrm{Mpc}^{-1}$. Galaxy cluster masses are given as $M_{500}$, the mass enclosed within $r_{500}$, within which the average density is 500 times the critical density of the universe at that redshift (e.g., Coe 2010). These masses $M_{500}$ are less than the total virial cluster masses measured at larger radii.

\section{Science}

RELICS was primarily designed and optimized to search for brightly lensed high-redshift galaxies in the epoch of reionization. Ancillary science enabled by RELICS includes supernova (SN) searches, cluster mass measurements, and limits on the dark matter particle cross section. We discuss each of these science goals in turn.

\subsection{High-redshift Galaxies}

HST's WFC3 has revealed $\sim 2000 z \sim 6-11$ candidates from the universe's first billion years, but only a small fraction have been bright enough $(H<25.5)$ for detailed follow-up study and spectroscopic confirmation (e.g., Bouwens et al. 2015; Salmon et al. 2017). RELICS was designed primarily to deliver (1) brightly observed high-redshift candidates, amenable to more detailed studies; and (2) a large sample of high-redshift candidates to improve luminosity function constraints. This combination is required to improve our understanding of galaxies and reionization in the first billion years.

\subsubsection{Spectroscopic Studies of Brightly Observed High-redshift Candidates}

Spectroscopic redshift confirmations based on Ly $\alpha$ detections have proven increasingly difficult beyond $z>6$ (Stark et al. 2010; Bradač et al. 2012; Schenker et al. 2014; Schmidt et al. 2016; Hoag et al. 2019; Mason et al. 2019), likely due primarily to absorption by patchy neutral hydrogen before reionization was complete (Treu et al. 2013; Tilvi et al. 2014;
Mason et al. 2018). A higher success rate was achieved with four luminous $z \sim 7-9$ galaxies inferred to have significant $[\mathrm{O}$ III $]+\mathrm{H} \beta$ equivalent width (EW) based on Spitzer photometry (Roberts-Borsani et al. 2016). All four yielded Ly $\alpha$ detections as distant as $z=8.68$ (Oesch et al. 2015; Zitrin et al. 2015b; Stark et al. 2017). This suggests that luminous galaxies with high ionization parameters carve out ionized gas bubbles allowing Ly $\alpha$ to stream free (Stark et al. 2017).

Alternatives to $\operatorname{Ly} \alpha$ redshift confirmations include fainter, slightly redder UV metal lines such as N V $\lambda 1243$, C IV $\lambda 1549$, He II $\lambda 1640$, O III] $\lambda 1663$, and C III] $\lambda 1908$ (Stark et al. 2014) and submillimeter lines such as [C II] $158 \mu \mathrm{m}$ and [O III] $88 \mu \mathrm{m}$ (Inoue et al. 2014). Studying these lines also yields more information about the physical properties of the galaxies, including their ionizing strength.

The UV metal line C III] has been detected in several $z>6$ galaxies as distant as $z=7.73$ (Stark et al. 2015, 2017; Laporte et al. 2017a; Mainali et al. 2018). At $z \sim 2$, Rigby et al. (2015) found that lower-metallicity galaxies exhibit stronger $\mathrm{C} \mathrm{III]}$, likely explaining why $\mathrm{C}$ III] appears far more often at $z \sim 6$ than at lower redshifts (see also Senchyna et al. 2017; Du et al. 2017; Le Fèvre et al. 2019). C III] detections may be powered by low-metallicity massive stars (Stark et al. 2015, 2017) or may require active galactic nuclei (AGNs; Nakajima et al. 2018). Still lower-metallicity stars $\left(<0.05 Z_{\odot}\right)$ produce higher ionization potentials yielding C IV. All three known $z>6 \mathrm{C}$ IV detections are lensed (Stark et al. 2015; Hoag et al. 2017; Mainali et al. 2017) and thus less massive and presumably lower metallicity than average $z>6$ galaxies from current surveys.

Larger samples of UV metal line observations including RELICS galaxies will enable us to quantify the prevalence of these intense ionizing sources in the reionization epoch, both directly and by extrapolating to larger samples with similar observed properties (luminosity, rest-frame UV slope $\beta$, and $\operatorname{Ly} \alpha$ ). The observed EWs will also be very informative to future JWST surveys planning to observe these spectral features.

A new window for studying high-redshift galaxies has been opened by the Atacama Large Millimeter/submillimeter Array (ALMA) in the south and the Plateau de Bure interferometer $(\mathrm{PdBI})$ in the north, later upgraded and renamed the Northern Extended Millimeter Array (NOEMA). These telescopes (primarily ALMA) have detected high-redshift galaxies by their infrared continuum dust emission (Capak et al. 2015), as well as spectral lines [C II] $158 \mu \mathrm{m}$ and [O III] $88 \mu \mathrm{m}$, which trace star formation (De Looze et al. 2014). Continuum observations show that $z \sim 5-6$ galaxies have at least an order of magnitude less dust than local starbursts with similar restframe UV colors (Capak et al. 2015). Dust is often not detected at these redshifts, but at least a few galaxies have been found to be dusty and thus evolved as early as $z=7.15$ (Hashimoto et al. 2019), $z=7.5$ (Watson et al. 2015), $z=8.312$ (Tamura et al. 2019), and $z=8.38$ (Laporte et al. 2017a).

[C II] $158 \mu \mathrm{m}$ is one of the brightest lines in local galaxies (Malhotra et al. 1997; Brauher et al. 2008) and the strongest observed interstellar medium cooling line in $z \sim 1-2$ galaxies (Stacey et al. 2010). To date, [C II] has been detected in 22 galaxies between $5.1533 \leqslant z \leqslant 7.1453$ (Carniani et al. 2018 and references therein; Hashimoto et al. 2019), including two spatially resolved galaxies at $z=6.81$ and 6.85 displaying disk-like rotation (Smit et al. 2018). 
Table 2

RELICS Clusters

\begin{tabular}{|c|c|c|c|c|c|c|c|}
\hline \multirow[b]{2}{*}{ Index } & \multirow[b]{2}{*}{ Cluster $^{\mathrm{a}}$} & \multirow{2}{*}{$\begin{array}{l}\text { R.A. }^{\mathrm{b}} \\
(\mathrm{J} 2000)\end{array}$} & \multirow{2}{*}{$\begin{array}{l}\text { Decl. }^{\mathrm{b}} \\
(\mathrm{J} 2000)\end{array}$} & \multirow[b]{2}{*}{ Redshift } & \multicolumn{2}{|c|}{ Planck Mass $M_{500}$} & \multirow[b]{2}{*}{$E(B-V)$} \\
\hline & & & & & $\operatorname{Rank}^{\mathrm{c}}$ & $\overline{\left(10^{14} M_{\odot}\right)}$ & \\
\hline \multirow[t]{2}{*}{1} & A2163 NE & $16: 15: 48.3$ & $-06: 07: 36.7$ & 0.203 & 1 & $16.12_{-0.29}^{+0.30}$ & 0.2972 \\
\hline & A2163 SW & $16: 15: 42.6$ & $-06: 09: 22.1$ & & & & \\
\hline 2 & PLCK G287.0+32.9 & $11: 50: 50.8$ & $-28: 04: 52.2$ & $0.390^{\mathrm{d}}$ & 2 & $14.69_{-0.42}^{+0.39}$ & 0.0813 \\
\hline 3 & MACS J0417.5-1154 & $04: 17: 33.7$ & $-11: 54: 22.6$ & 0.443 & 4 & $12.25_{-0.55}^{+0.53}$ & 0.0320 \\
\hline 4 & A697 & $08: 42: 58.9$ & $+36: 21: 51.1$ & 0.282 & 10 & $11.00_{-0.37}^{+0.37}$ & 0.0333 \\
\hline \multirow[t]{2}{*}{5} & RXS J060313.4+4212 N & $06: 03: 12.2$ & $+42: 15: 24.7$ & 0.228 & 11 & $10.76_{-0.43}^{+0.45}$ & 0.1933 \\
\hline & RXS J060313.4+4212 S & $06: 03: 25.6$ & $+42: 09: 53.6$ & & & & \\
\hline 6 & MACS J0308.9+2645 & 03:08:55.7 & $+26: 45: 36.8$ & 0.356 & 12 & $10.76_{-0.65}^{+0.63}$ & 0.1776 \\
\hline \multirow[t]{2}{*}{7} & ACT-CL J0102-49151 NW & 01:02:53.1 & $-49: 14: 52.8$ & 0.870 & 13 & $10.75_{-0.47}^{+0.48}$ & 0.0086 \\
\hline & ACT-CL J0102-49151 SE & 01:03:00.0 & $-49: 16: 22.2$ & & & & \\
\hline 8 & RXC J0600.1-2007 & 06:00:09.8 & $-20: 08: 08.9$ & 0.460 & 14 & $10.73_{-0.54}^{+0.51}$ & 0.0433 \\
\hline 9 & PSZ2 G209.79+10.23 & $07: 22: 23.0$ & $+07: 24: 30.0$ & 0.677 & 15 & $10.73_{-0.66}^{+0.63}$ & 0.0375 \\
\hline 10 & PLCK G171.9-40.7 & $03: 12: 56.9$ & $+08: 22: 19.2$ & 0.270 & 16 & $10.71_{-0.50}^{+0.49}$ & 0.4477 \\
\hline 11 & RXC J2211.7-0350 & $22: 11: 45.9$ & $-03: 49: 44.7$ & 0.397 & 17 & $10.50_{-0.49}^{+0.50}$ & 0.0832 \\
\hline 12 & PLCK G004.5-19.5 & $19: 17: 04.5$ & $-33: 31: 28.5$ & $0.519^{\mathrm{e}}$ & 19 & $10.36_{-0.72}^{+0.68}$ & 0.0790 \\
\hline 13 & PLCK G308.3-20.2 & $15: 18: 49.9$ & $-81: 30: 33.6$ & 0.480 & 20 & $10.32_{-0.58}^{+0.57}$ & 0.2348 \\
\hline 14 & RXC J0018.5+1626 & $00: 18: 32.6$ & $+16: 26: 08.4$ & 0.546 & 24 & $9.79_{-0.53}^{+0.53}$ & 0.0501 \\
\hline 15 & SPT-CL J0254-5857 & $02: 54: 16.0$ & $-58: 57: 11.0$ & 0.438 & 26 & $9.69_{-0.38}^{+0.37}$ & 0.0183 \\
\hline 16 & PSZ2 G138.61-10.84 & $02: 27: 06.6$ & $+49: 00: 29.9$ & 0.702 & 27 & $9.48_{-0.53}^{+0.67}$ & 0.1830 \\
\hline 17 & RXC J0142.9+4438 & $01: 42: 55.2$ & $+44: 38: 04.3$ & 0.341 & 28 & $9.02_{-0.64}^{+0.60}$ & 0.0783 \\
\hline 18 & A1300 & $11: 31: 54.1$ & $-19: 55: 23.4$ & 0.308 & 30 & $8.97_{-0.45}^{+0.46}$ & 0.0440 \\
\hline 19 & WHL J013719.8-082841 & $01: 37: 25.0$ & $-08: 27: 25.0$ & 0.566 & 31 & $8.93_{-0.70}^{+0.65}$ & 0.0286 \\
\hline 20 & RXC J1514.9-1523 & $15: 15: 00.7$ & $-15: 22: 46.7$ & 0.223 & 33 & $8.86_{-0.46}^{+0.41}$ & 0.0869 \\
\hline 21 & A665 & $08: 30: 57.4$ & $+65: 50: 31.0$ & 0.182 & 34 & $8.86_{-0.32}^{+0.32}$ & 0.0400 \\
\hline 22 & MACS J0553.4-3342 & $05: 53: 23.1$ & $-33: 42: 29.9$ & 0.430 & 36 & $8.77_{-0.46}^{+0.44}$ & 0.0357 \\
\hline 23 & SMACS J0723.3-7327 & $07: 23: 19.5$ & $-73: 27: 15.6$ & 0.390 & 43 & $8.39_{-0.34}^{+0.33}$ & 0.1893 \\
\hline 24 & RXC J0949.8+1707 & $09: 49: 50.9$ & $+17: 07: 15.3$ & 0.383 & 48 & $8.24_{-0.46}^{+0.46}$ & 0.0255 \\
\hline \multirow[t]{2}{*}{25} & A1758a NW & $13: 32: 39.0$ & $+50: 33: 41.8$ & 0.280 & 50 & $8.22_{-0.28}^{+0.27}$ & 0.0122 \\
\hline & A1758a SE & $13: 32: 53.4$ & $+50: 31: 31.0$ & & & & \\
\hline 26 & A1763 & $13: 35: 18.9$ & $+40: 59: 57.2$ & 0.228 & 51 & $8.13_{-0.27}^{+0.26}$ & 0.0073 \\
\hline 27 & A2813 & $00: 43: 25.1$ & $-20: 37: 14.8$ & 0.292 & 52 & $8.13_{-0.38}^{+0.37}$ & 0.0178 \\
\hline \multirow[t]{2}{*}{28} & A520 NE & $04: 54: 19.0$ & $+02: 56: 49.0$ & 0.203 & 65 & $7.80_{-0.41}^{+0.40}$ & 0.0402 \\
\hline & A520 SW & 04:54:04.2 & $+02: 53: 41.9$ & & & & \\
\hline 29 & RXC J0032.1+1808 & $00: 32: 11.0$ & $+18: 07: 49.0$ & 0.396 & 85 & $7.61_{-0.63}^{+0.57}$ & 0.1052 \\
\hline 30 & RXC J0232.2-4420 & $02: 32: 18.1$ & $-44: 20: 44.9$ & 0.284 & 91 & $7.54_{-0.32}^{+0.33}$ & 0.0165 \\
\hline 31 & A3192 & 03:58:53.1 & $-29: 55: 44.8$ & 0.425 & 114 & $7.20_{-0.50}^{+0.52}$ & 0.0071 \\
\hline 32 & MACS J0159.8-0849 & $01: 59: 49.4$ & $-08: 50: 00.0$ & 0.405 & 115 & $7.20_{-0.68}^{+0.61}$ & 0.0207 \\
\hline 33 & MACS J0035.4-2015 & $00: 35: 27.0$ & $-20: 15: 40.3$ & 0.352 & 133 & $7.01_{-0.50}^{+0.45}$ & 0.0187 \\
\hline 34 & RXC J0911.1+1746 & 09:11:11.4 & $+17: 46: 33.5$ & 0.505 & 136 & $6.99_{-0.79}^{+0.73}$ & 0.0359 \\
\hline 35 & AS295 & $02: 45: 31.4$ & $-53: 02: 24.9$ & 0.300 & 156 & $6.78_{-0.36}^{+0.37}$ & 0.0445 \\
\hline 36 & SPT-CL J0615-5746 & $06: 15: 54.2$ & $-57: 46: 57.9$ & 0.972 & 157 & $6.77_{-0.54}^{+0.49}$ & 0.0362 \\
\hline 37 & MACS J0257.1-2325 & $02: 57: 10.2$ & $-23: 26: 11.8$ & 0.505 & 227 & $6.22_{-0.74}^{+0.70}$ & 0.0251 \\
\hline 38 & A2537 & $23: 08: 22.2$ & $-02: 11: 32.4$ & 0.297 & 376 & $5.52_{-0.51}^{+0.51}$ & 0.0798 \\
\hline 39 & MS 1008.1-1224 & $10: 10: 33.6$ & $-12: 39: 43.0$ & 0.306 & 504 & $4.94_{-0.60}^{+0.57}$ & 0.0601 \\
\hline 40 & MACS J0025.4-1222 & $00: 25: 30.3$ & $-12: 22: 48.1$ & 0.586 & $\cdots$ & $\ldots$ & 0.0239 \\
\hline 41 & CL J0152.7-1357 & 01:52:42.9 & $-13: 57: 31.0$ & 0.833 & $\ldots$ & $\ldots$ & 0.0126 \\
\hline
\end{tabular}

Notes.

${ }^{\text {a }}$ Cluster name and portion (e.g., NE) if observed by RELICS with two WFC3/IR pointings.

${ }^{\mathrm{b}}$ Coordinates of $H S T$ WFC3/IR pointings.

${ }^{\mathrm{c}}$ Mass rank among all 1094 clusters in the PSZ2 catalog.

${ }^{\mathrm{d}}$ Redshift updated in Zitrin et al. (2017); previously $z=0.38$.

${ }^{\mathrm{e}}$ Redshift updated in Albert et al. (2017); previously $z=0.54$.

${ }^{\mathrm{f}}$ Observations of MACS J0358.8-2955 $(z=0.428)$ include contributions from A3192 $(z=0.168)$.

At higher redshifts, Inoue et al. (2014) predicted that [O III] $88 \mu \mathrm{m}$ would yield more detections. While [C II] is associated with neutral $\mathrm{H}$ I gas in photodissociation regions (PDRs), [O III] is associated with ionized $\mathrm{H}$ II gas, more prevalent in higherredshift, lower-metallicity galaxies with higher ionization states (Harikane et al. 2018). As predicted, ALMA's highest-redshift 
Table 3

RELICS Cluster Names

\begin{tabular}{|c|c|c|c|c|}
\hline Index & Cluster & Alternate Name & Abbreviation & Notes \\
\hline 1 & A2163 & & abell2163 & \\
\hline 2 & PLCK G287.0+32.9 & & plckg287+32 & \\
\hline 3 & MACS J0417.5-1154 & & $\operatorname{macs} 0417-11$ & (a) \\
\hline 4 & A697 & & abell697 & \\
\hline 5 & RXS J060313.4+4212 & "Toothbrush" & rxs0603+42 & \\
\hline 6 & MACS J0308.9+2645 & & $\operatorname{macs} 0308+26$ & \\
\hline 7 & ACT-CL J0102-49151 & "El Gordo" & act0102-49 & (b) \\
\hline 8 & RXC J0600.1-2007 & MACS J0600.1-2007 & & \\
\hline 9 & PSZ2 G209.79+10.23 & & plckg209+10 & \\
\hline 10 & PLCK G171.9-40.7 & & plckg171-40 & \\
\hline 11 & RXC J2211.7-0350 & MACS J2211.7-0350 & rxc2211-03 & \\
\hline 12 & PLCK G004.5-19.5 & & plckg004-19 & \\
\hline 13 & PLCK G308.3-20.2 & SMACS J1519.1-8130 & plckg308-20 & \\
\hline 14 & RXC J0018.5+1626 & MS0015.9+1609 MACS J0018.5+1626 & $\operatorname{rxc} 0018+16$ & \\
\hline 15 & SPT-CL J0254-5857 & & spt0254-58 & \\
\hline 16 & PSZ2 G138.61-10.84 & & plckg138-10 & (c) \\
\hline 17 & RXC J0142.9+4438 & CIZA J0142.9+4438 & $\mathrm{rxc} 0142+44$ & \\
\hline 18 & A1300 & & abell1300 & \\
\hline 19 & WHL J013719.8-082841 & WHL J24.3324-8.477 & whl0137-08 & (d) \\
\hline 20 & RXC J1514.9-1523 & & rxc1514-15 & \\
\hline 21 & A665 & & abell665 & \\
\hline 22 & MACS J0553.4-3342 & & macs0553-33 & \\
\hline 23 & SMACS J0723.3-7327 & & smacs0723-73 & \\
\hline 24 & RXC J0949.8+1707 & & rxc0949+17 & (e) \\
\hline 25 & A1758a & & abell1758 & \\
\hline 26 & A1763 & & abell1763 & \\
\hline 27 & A2813 & & abell2813 & \\
\hline 28 & A520 & "Train Wreck" & abell520 & \\
\hline 29 & RXC J0032.1+1808 & & $\mathrm{rxc} 0032+18$ & \\
\hline 30 & RXC J0232.2-4420 & "RBS-0325" & rxc0232-44 & \\
\hline 31 & A3192 & MACS J0358.8-2955 & abell3192 & (f) \\
\hline 32 & MACS J0159.8-0849 & & $\operatorname{macs} 0159-08$ & (g) \\
\hline 33 & MACS J0035.4-2015 & & $\operatorname{macs} 0035-20$ & \\
\hline 34 & RXC J0911.1+1746 & MACS J0911.1+1746 & $\operatorname{rxc} 0911+17$ & \\
\hline 35 & AS295 & ACT-CL J0245-5302 SPT-CL J0245-5302 & abells295 & \\
\hline 36 & SPT-CL J0615-5746 & & spt0615-57 & \\
\hline 37 & MACS J0257.1-2325 & & $\operatorname{macs} 0257-23$ & (h) \\
\hline 38 & A2537 & & abell2537 & (h) \\
\hline 39 & MS 1008.1-1224 & & ms $1008-12$ & \\
\hline 40 & MACS J0025.4-1222 & "Baby Bullet" & $\operatorname{macs} 0025-12$ & \\
\hline 41 & CL J0152.7-1357 & & $\operatorname{cl0152-13}$ & \\
\hline
\end{tabular}

Notes.

a JWST GTO target (CANUCS; PI Willott).

b JWST GTO target (PI Windhorst).

${ }^{c}$ Optical counterpart is 4'.5 from PSZ1 coordinates (2:27:06, +49:05:10) from Planck Collaboration et al. (2016d).

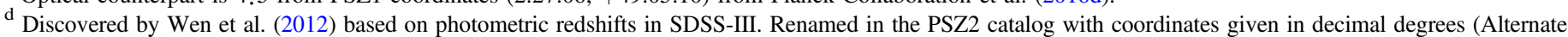
Name above).

' Wong et al. (2013) \#7: seventh most powerful lensing line of sight expected based on LRGs identified in SDSS.

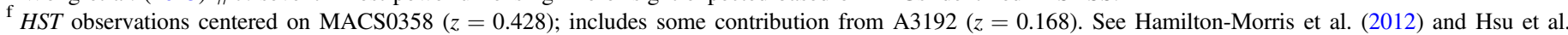
(2013).

g Wong et al. (2013) \#59.

${ }^{\mathrm{h}}$ Frontier Fields contender (ultimately not selected).

spectroscopic confirmations have come from [O III], and the six $z>6$ galaxies targeted to date have all yielded [O III] detections at $z=6.900$ (Marrone et al. 2018), $z=7.107$ (Carniani et al. 2017), $z=7.212$ (Inoue et al. 2016), $z=8.312$ (Tamura et al. 2019), $z=8.382$ (Laporte et al. 2017b), and $z=9.11$ (Hashimoto et al. 2018). The two highest-redshift detections are lensed galaxies.

Based on this previous work, we expect RELICS to deliver ALMA confirmations and science at $z \sim 6-10$ (see Section 6.1).

\subsubsection{Luminosity Functions of Galaxies at the Epoch of Reionization}

Improving constraints on the $z \sim 9$ luminosity function (and the evolution from $z \sim 10$ to 6 ) is another primary science goal of RELICS. This is required to determine the numbers of faint galaxies available to reionize the universe.

Planck constrained the reionization history by measuring the column density of free electrons to the $\mathrm{CMB}$, or the Thomson scattering optical depth $\tau=0.058 \pm 0.012$ (Planck Collaboration et al. 2018a). This value, lower than previous estimates, 


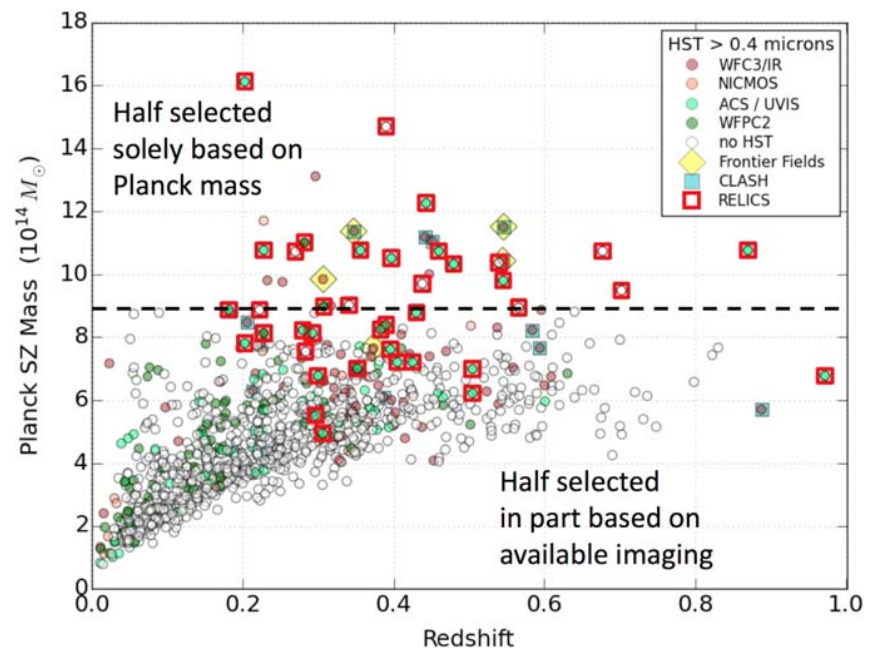

Figure 1. RELICS clusters marked as red squares on a plot of mass vs. redshift for the 1094 clusters in the Planck PSZ2 catalog. Each cluster is plotted as a circle color-coded according to existing HST imaging prior to RELICS, prioritizing WFC3/IR (red) followed by NICMOS (salmon), ACS and/or UVIS (aqua), and finally WFPC2 (green). Clusters without HST imaging prior to RELICS are colored white. Frontier Fields and CLASH clusters are plotted as filled yellow diamonds and blue squares, respectively. A dashed line at $8.7 \times 10^{14} M_{\odot}$ separates the 21 clusters selected solely by mass from the other 20 selected based on various criteria, including existing imaging revealing lensing strength. Note that two RELICS clusters are not plotted here, as they were not included in the Planck PSZ2 catalog.

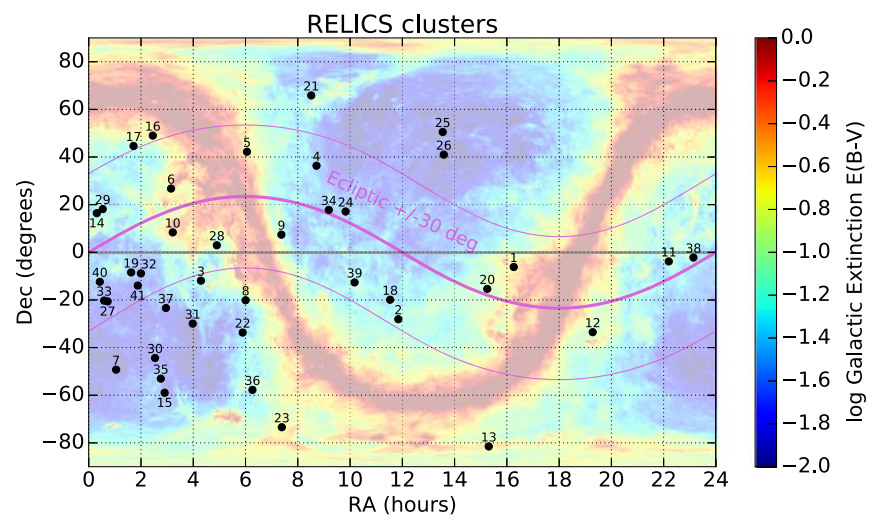

Figure 2. Distribution on the sky of the 41 RELICS clusters relative to the Galactic and ecliptic planes. The background is color-coded to show the Galactic extinction map from Schlegel et al. (1998). Numbers correspond to ordered Planck mass as given in Table 2.

implies a later reionization halfway complete by $z \sim 8$, before fully completing by $z \sim 6$. It also means that reionization can be achieved by galaxies producing less Lyman continuum (LyC) flux and with lower escape fractions $f_{\text {esc }}$. Direct measurements of these $f_{\text {esc }}$ values have been obtained recently for $\sim 40$ galaxies with detected LyC leakage, including half extending from the local universe (e.g., Leitherer et al. 2016; Puschnig et al. 2017) to $z \sim 0.3$ (e.g., Izotov et al. 2018), with the other half at $z \sim 2-4$ (e.g., Shapley et al. 2016; RiveraThorsen et al. 2017; Vanzella et al. 2018). Assuming that these measurements hold at higher redshifts, and given high-redshift luminosity functions, low-mass galaxies could have produced most of the flux required to reionize the universe (e.g., Robertson et al. 2015; Madau 2017; Ishigaki et al. 2018; Mason et al. 2018; Finkelstein et al. 2019). There may also have been significant contributions from low-luminosity AGN jets at $z \gtrsim 6$ (Bosch-Ramon 2018).

At the highest redshifts $(z \sim 9-12)$, HST and Spitzer imaging programs have yielded fewer candidates than expected. This has left luminosity functions highly uncertain at these redshifts, while also hinting at accelerated evolution in the first $600 \mathrm{Myr}$ (Bouwens et al. 2012; Oesch et al. 2018). Luminosity functions are fairly well constrained at $z \sim 4-8$ (Bouwens et al. 2015; Finkelstein 2016; Kawamata et al. 2018). Only recently were constraints placed on all three parameters $\left(\phi^{*}, M^{*}, \alpha\right)$ of the $z \sim 9$ luminosity function (Morishita et al. 2018); these results are consistent with either accelerated or smoother (nonaccelerated) evolution at $z>8$. Higher-precision measurements, especially with greater leverage at higher redshifts, are required to better constrain this early evolution rate.

\subsection{Strong-lens Modeling}

Robust strong-lens modeling is required to estimate magnifications of our lensed galaxies and the survey volume. The mass in galaxy cluster cores responsible for the lensing is predominantly dark matter. We must infer the distribution of this dark matter based on observed strong lensing that produces multiple images of more distant galaxies. We often add the assumption that luminous galaxy cluster members are good tracers of their dark matter halos.

High-quality lens modeling generally requires multiband highresolution $H S T$ imaging to reliably identify multiple images of strongly lensed galaxies based on their colors and morphologies. Spectroscopic redshifts are also crucial. Magnification accuracies improve with greater numbers of strongly lensed images with spectroscopic redshifts (Johnson \& Sharon 2016). Recent tests with simulated cluster lensing find that the best lens models accurately yield magnification estimates of 3 (10) with precisions of $15 \%(30 \%)$, with uncertainty increasing with magnification (Meneghetti et al. 2017; see also Zitrin et al. 2015a). Such precision is encouraging, as the vast majority (over 80\%) of lensed high-redshift galaxies observed are magnified by factors of 10 or less (Coe et al. 2015).

Magnification uncertainties directly impact measurements of some physical properties such as luminosity, star formation rate, stellar mass, and size. However, since lensing is achromatic, other properties derived from galaxy colors are not affected by magnification; these include redshift, age, metallicity, extinction, and rest-frame UV slope. Magnifications do affect luminosity functions, but the uncertainties are mitigated by averaging over many galaxies. The resulting uncertainties on the total survey volume are subdominant compared to the current small number statistics at high redshifts (Coe et al. 2015).

In the case of a multiply imaged high-redshift candidate, lens modeling can yield geometric support, distinguishing between low- and high-redshift solutions based on the separation between the observed images (e.g., Coe et al. 2013; Zitrin et al. 2014; Chan et al. 2017). Lens modeling is required to study delensed (source plane) properties, including $\sim 100 \mathrm{pc}$ structures resolved in highly elongated arcs (e.g., Sharon et al. 2012; Johnson et al. 2017). Lens modeling is also required to constrain luminosity functions in lensed fields (e.g., Livermore et al. 2017).

RELICS lens modeling is yielding the overall lensing strength for each cluster. While we expect all RELICS clusters to be excellent lenses, our analyses will reveal which are truly among the strongest and best to use for efficient discoveries of 
Table 4

Archival HST ACS and WFC3/UVIS Imaging of RELICS Clusters

\begin{tabular}{|c|c|c|c|c|c|c|c|c|c|c|}
\hline $\begin{array}{l}\text { Cluster } \\
\text { Index }\end{array}$ & $\begin{array}{l}\text { Abbreviated } \\
\text { Name }^{\mathrm{a}}\end{array}$ & $\begin{array}{c}\text { F390W } \\
\text { (s) }\end{array}$ & $\begin{array}{c}\text { F435W } \\
\text { (s) }\end{array}$ & $\begin{array}{c}\mathrm{F} 475 \mathrm{~W} \\
\text { (s) }\end{array}$ & $\begin{array}{c}\text { F555W } \\
\text { (s) }\end{array}$ & $\begin{array}{c}\text { F606W } \\
\text { (s) }\end{array}$ & $\begin{array}{c}\text { F625W } \\
\text { (s) }\end{array}$ & $\begin{array}{c}\text { F775W } \\
\text { (s) }\end{array}$ & $\begin{array}{c}\text { F814W } \\
\text { (s) }\end{array}$ & $\begin{array}{c}\text { F850LP } \\
\text { (s) }\end{array}$ \\
\hline 1 & abell2163 & & $4664^{b}$ & & & $4667^{b}$ & & & $9192^{b}$ & \\
\hline 2 & plckg $287+32$ & & & 2160 & & 2320 & & & 4680 & \\
\hline 3 & macs0417-11 & & & & & $7152^{c}$ & & & 1910 & \\
\hline 4 & abell697 & & & & & & & & & \\
\hline 5 & rxs0603+42 & $5294^{\mathrm{b}, \mathrm{c}}$ & & & & $5068^{\mathrm{b}, \mathrm{c}}$ & & & $10194^{\mathrm{b}}$ & \\
\hline 6 & $\operatorname{macs} 0308+26$ & & & & & 1200 & & & 1440 & \\
\hline 7 & act0102-49 & & & & & $7680^{\mathrm{b}}$ & $4688^{\mathrm{b}}$ & $5024^{\mathrm{b}}$ & 1916 & $5032^{\mathrm{b}}$ \\
\hline 8 & rxc0600-20 & & & & & & & & 1440 & \\
\hline 9 & plckg $209+10$ & & & & & & & & & \\
\hline 10 & plckg171-40 & & & & & & & & & \\
\hline 11 & rxc2211-03 & & & & & 1200 & & & & \\
\hline 12 & plckg004-19 & & & & & & & & & \\
\hline 13 & plckg308-20 & & & & & 1200 & & & & \\
\hline 14 & rxc0018+16 & & & & 4500 & $17920^{\mathrm{b}}$ & & 4623 & 4560 & 2540 \\
\hline 15 & spt0254-58 & & & & & & & & & \\
\hline 16 & plckg138-10 & & & & & & & & & \\
\hline 17 & rxc0142+44 & & & & & & & & & \\
\hline 18 & abell1300 & & & & & & & & & \\
\hline 19 & whl0137-08 & & & & & & & & & \\
\hline 20 & rxc1514-15 & & & & & & & & & \\
\hline 21 & abell665 & & & & & & 2680 & & & \\
\hline 22 & macs0553-33 & & 4452 & & & 2092 & & & 4572 & \\
\hline 23 & smacs0723-73 & & & & & 1200 & & & 1440 & \\
\hline 24 & rxc0949+17 & & & & & 1200 & & & 1440 & \\
\hline 25 & abell1758 & & $5072^{\mathrm{b}}$ & & & $5088^{\mathrm{b}}$ & & & $10000^{\mathrm{b}}$ & \\
\hline 26 & abell1763 & & & & & & & & & \\
\hline 27 & abell2813 & & & & & 1200 & & & & \\
\hline 28 & abell520 & & $9296^{\mathrm{b}}$ & & & $9328^{\mathrm{b}}$ & & & $18320^{\mathrm{b}}$ & \\
\hline 29 & $\operatorname{rxc} 0032+18$ & & & & & 1200 & & & 1440 & \\
\hline 30 & rxc0232-44 & & & & & & & & & \\
\hline 31 & abell3192 & & 4500 & & & 3320 & & & 4620 & \\
\hline 32 & $\operatorname{macs} 0159-08$ & & & & & 1200 & & & & \\
\hline 33 & $\operatorname{macs} 0035-20$ & & & & & 1200 & & & 1440 & \\
\hline 34 & rxc0911+17 & & & & 4470 & & & & 8825 & \\
\hline 35 & abells295 & & 1920 & & & 1936 & & & 3944 & \\
\hline 36 & spt0615-57 & & & & & $8880^{\mathrm{b}}$ & & & $12720^{\mathrm{d}}$ & \\
\hline 37 & $\operatorname{macs} 0257-23$ & & & & 4500 & & & & 8858 & \\
\hline 38 & abell2537 & & & & & 2080 & & & & \\
\hline 39 & $\mathrm{~ms} 1008-12$ & & & & & $17856^{\mathrm{b}}$ & & 2440 & & 2560 \\
\hline 40 & $\operatorname{macs} 0025-12$ & & & & 4140 & & & & 4200 & \\
\hline 41 & cl0152-13 & & & & & & $19000^{\mathrm{b}}$ & $23452^{d}$ & & $19000^{\mathrm{b}}$ \\
\hline
\end{tabular}

Notes.

${ }^{\text {a }}$ Abbreviated names provided here are used elsewhere in the text and in data product filenames.

${ }^{b}$ Multiple pointings with minimal or no overlap are given in superscript brackets. Total exposure times listed should be divided by the numbers [2] or [4] to give the depth at each position.

${ }^{c}$ WFC3/UVIS observations. (All other archival observations listed are ACS.)

d $2 \times 2$ mosaic plus one extra central pointing; dividing that exposure time by 2.5 yields $5088 \mathrm{~s}$ within the central pointing.

the most distant galaxies known in future surveys. For RELICS results to date, see Section 6.2.

\subsection{Galaxy Cluster Masses}

Scaling relations linking mass estimates from lensing, X-ray, and SZ studies require understanding of observational systematics and scale-dependent cluster astrophysics. Improving the accuracy of these mass scaling relations will be key to realizing the full potential of future missions such as eROSITA, which anticipates detecting 100,000 clusters/groups out to $z \sim 1.3$ (Pillepich et al. 2018).
Constraints on cosmological parameters (primarily $\Omega_{m}$ and $\sigma_{8}$ ) derived from the Planck CMB (Planck Collaboration et al. 2016b) are at odds with those derived from Planck SZ galaxy cluster counts (Planck Collaboration et al. 2016c). The latter paper calibrated the scaling relation between Planck SZ signal strength and cluster masses based on analyses of XMM-Newton $\mathrm{X}$-ray observations assuming hydrostatic equilibrium (HSE). One may expect cluster masses to be $\sim 20 \%$ greater owing to deviations from HSE, including nonthermal pressure support (e.g., Nagai et al. 2007; Rasia et al. 2012). But Planck Collaboration et al. (2016c) found that the cosmology results can be reconciled only if one assumes that SZ-derived cluster 
Table 5

Archival HST Imaging Programs of RELICS Clusters

\begin{tabular}{|c|c|c|c|c|}
\hline $\begin{array}{l}\text { Cluster } \\
\text { Index }\end{array}$ & $\begin{array}{l}\text { Abbreviated } \\
\text { Name }\end{array}$ & $\begin{array}{l}\text { Program } \\
\text { ID }\end{array}$ & $\begin{array}{l}\text { Principal } \\
\text { Investigator }\end{array}$ & Filters \\
\hline 1 & abell2163 & 12253 & Clowe & $\begin{array}{l}\mathrm{F} 435 \mathrm{~W}^{\mathrm{a}}, \mathrm{F} 606 \mathrm{~W}^{\mathrm{a}}, \\
\mathrm{F}^{2} 14 \mathrm{~W}^{\mathrm{a}}\end{array}$ \\
\hline 2 & plckg287+32 & 14165 & Seitz & $\begin{array}{l}\text { F475W, F606W, } \\
\text { F814W, } \\
\text { F110W }\end{array}$ \\
\hline 3 & $\operatorname{macs} 0417-11$ & 12009 & von der Linden & F606W $W^{\mathrm{c}}, \mathrm{F} 814 \mathrm{~W}$ \\
\hline 5 & rxs0603+42 & 13343 & Wittman & $\begin{array}{l}\mathrm{F} 390 \mathrm{~W}^{\mathrm{a}, \mathrm{c}}, \mathrm{F} 606 \mathrm{~W}^{\mathrm{a}}, \\
\quad{ }^{\mathrm{c}}, \mathrm{F} 814 \mathrm{~W}^{\mathrm{a}}\end{array}$ \\
\hline \multirow[t]{2}{*}{6} & \multirow[t]{2}{*}{$\operatorname{macs} 0308+26$} & 12166 & Ebeling & F606W \\
\hline & & 12884 & Ebeling & F814W \\
\hline \multirow[t]{2}{*}{7} & \multirow[t]{2}{*}{ act0102-49 } & 12477 & High & F606W ${ }^{\mathrm{a}}, \mathrm{F} 814 \mathrm{~W}$ \\
\hline & & 12755 & Hughes & $\begin{array}{l}\mathrm{F}^{2} 25 \mathrm{~W}^{\mathrm{a}}, \mathrm{F} 775 \mathrm{~W}^{\mathrm{a}}, \\
\mathrm{F}^{2} 50 \mathrm{LP}^{\mathrm{a}}\end{array}$ \\
\hline 8 & rxc0600-20 & 12884 & Ebeling & F814W \\
\hline 11 & rxc2211-03 & 12166 & Ebeling & F606W \\
\hline 13 & plckg308-20 & 12884 & Ebeling & F606W \\
\hline \multirow[t]{4}{*}{14} & \multirow[t]{4}{*}{ rxc0018+16 } & 9292 & Ford & F775W, F850LP \\
\hline & & 10493 & Gal-Yam & F775W \\
\hline & & 10635 & Ziegler & F606W ${ }^{\mathrm{a}}$ \\
\hline & & 11560 & Ebeling & F555W, F814W \\
\hline 21 & abell665 & 9292 & Ford & F625W \\
\hline 22 & $\operatorname{macs} 0553-33$ & 12362 & Ebeling & $\begin{array}{l}\text { F435W, F606W, } \\
\text { F814W }\end{array}$ \\
\hline \multirow[t]{2}{*}{23} & \multirow[t]{2}{*}{ smacs0723-73 } & 12166 & Ebeling & F606W \\
\hline & & 12884 & Ebeling & F814W \\
\hline \multirow[t]{2}{*}{24} & \multirow[t]{2}{*}{ rxc0949+17 } & 10491 & Ebeling & F606W \\
\hline & & 12166 & Ebeling & F814W \\
\hline 25 & abell1758 & 12253 & Clowe & $\begin{array}{c}\mathrm{F} 435 \mathrm{~W}^{\mathrm{a}}, \mathrm{F} 606 \mathrm{~W}^{\mathrm{a}}, \\
\mathrm{F}^{2} 14 \mathrm{~W}^{\mathrm{a}}\end{array}$ \\
\hline 27 & abell2813 & 10881 & Smith & F606W \\
\hline 28 & abell520 & 12253 & Clowe & $\begin{array}{c}\mathrm{F} 435 \mathrm{~W}^{\mathrm{a}}, \mathrm{F} 606 \mathrm{~W}^{\mathrm{a}}, \\
\mathrm{F}^{2} 14 \mathrm{~W}^{\mathrm{a}}\end{array}$ \\
\hline 29 & rxc0032+18 & 12166 & Ebeling & F606W, F814W \\
\hline \multirow[t]{2}{*}{31} & \multirow[t]{2}{*}{ abell3192 } & 10881 & Smith & F606W \\
\hline & & 12313 & Ebeling & $\begin{array}{l}\text { F435W, F606W, } \\
\text { F814W }\end{array}$ \\
\hline 32 & macs0159-08 & 12166 & Ebeling & F606W \\
\hline \multirow[t]{2}{*}{33} & \multirow[t]{2}{*}{$\operatorname{macs} 0035-20$} & 10491 & Ebeling & F606W \\
\hline & & 12884 & Ebeling & F814W \\
\hline \multirow[t]{3}{*}{34} & \multirow[t]{3}{*}{$\operatorname{rxc} 0911+17$} & 9722 & Ebeling & F555W, F814W \\
\hline & & 10493 & Gal-Yam & F814W \\
\hline & & 10793 & Gal-Yam & F814W \\
\hline 35 & abells295 & 13514 & Pacaud & $\begin{array}{l}\text { F435W, F606W, } \\
\text { F814W }\end{array}$ \\
\hline \multirow[t]{2}{*}{36} & \multirow[t]{2}{*}{ spt0615-57 } & 12477 & High & F606W ${ }^{\mathrm{a}}, \mathrm{F} 814 \mathrm{~W}$ \\
\hline & & 12757 & Mazzotta & $\mathrm{F} 814 \mathrm{~W}^{\mathrm{a}}$ \\
\hline \multirow[t]{3}{*}{37} & \multirow[t]{3}{*}{$\operatorname{macs} 0257-23$} & 9722 & Ebeling & F555W, F814W \\
\hline & & 10493 & Gal-Yam & F814W \\
\hline & & 10793 & Gal-Yam & F814W \\
\hline 38 & abell2537 & 9270 & Allen & F606W \\
\hline \multirow[t]{2}{*}{39} & \multirow[t]{2}{*}{ ms $1008-12$} & 9292 & Ford & F775W, F850LP \\
\hline & & 10635 & Ziegler & F606W \\
\hline 40 & $\operatorname{macs} 0025-12$ & 10703 & Ebeling & F555W, F814W \\
\hline \multirow[t]{3}{*}{41} & cl0152-13 & 9290 & Ford & $\begin{array}{l}\mathrm{F}^{2} 25 \mathrm{~W}^{\mathrm{a}}, \mathrm{F} 775 \mathrm{~W}^{\mathrm{a}}, \\
\mathrm{F}^{2} 50 \mathrm{LP}^{\mathrm{a}}\end{array}$ \\
\hline & & 10493 & Gal-Yam & F775W \\
\hline & & 10793 & Gal-Yam & F775W \\
\hline
\end{tabular}

\footnotetext{
Notes.

aultiple pointings with minimal or no overlap are given in superscript brackets.

b WFC3/IR observations obtained during the same HST Cycle as RELICS.

${ }^{c}$ WFC3/UVIS observations. (Other observations obtained prior to RELICS are all ACS.)
}

masses underestimate the true $M_{500}$ masses by $b \approx 42 \%$ (where they reported the mass bias as $1-b=0.58 \pm 0.04$ ). That is about $2 \sigma$ greater than the $\sim 20 \%$ initially adopted in the PSZ1 analysis (Planck Collaboration et al. 2014).

Weak-lensing analysis of clusters included in both the Planck analysis and the Weighing the Giants survey (WtG; von der Linden et al. 2014b) shows that Planck cluster masses may indeed be underestimated by $\sim 42 \%$ for the most massive clusters $\left(>10^{15} M_{\odot}\right)$, while Planck masses appear to be more accurate for less massive clusters $\left(\sim 5 \times 10^{14} M_{\odot}\right)$. Subsequent weak-lensing analyses from various surveys (WtG, CCCP, LoCUSS, CLASH, CFHTLenS, RCSLenS, HSC-SSP) found a range of results, some consistent with $\mathrm{WtG}$ including bias increasing with mass (Sereno \& Ettori 2017), and others more consistent with the original Planck estimate of 20\% bias (Hoekstra et al. 2015; Battaglia et al. 2016; Smith et al. 2016; Penna-Lima et al. 2017; Medezinski et al. 2018). Overall, the tension appears to be somewhat relieved, although not conclusively (Planck Collaboration et al. 2018b), especially after accounting for new Planck measurements of the reionization optical depth (Planck Collaboration et al. 2016e, 2018a).

SZ mass estimates are calibrated in part based on X-ray temperatures measured with spectroscopy out to $r_{500}$, which can be difficult, especially for higher-redshift clusters. X-ray mass calibration at the smaller $r_{2500}$ radii probed by HST strong lensing may be key to improving mass scaling relations. These smaller radii are also important for ground-based SZ telescopes offering significantly higher resolution than Planck. Czakon et al. (2015) analyzed arcsecond-resolution Bolocam SZ imaging of 45 clusters and found that the $Y_{\mathrm{SZ}}$ signal does not scale self-similarly with $M_{2500}$ derived from X-ray observations. The cause of this mismatch may be due to AGN feedback regulating star formation and altering the gas properties in cluster cores. Through accurate lensing mass profiles measuring total mass, free from the assumption of gas HSE, and accurate gas mass profiles from Chandra, we will determine the radial dependence of $f_{\mathrm{gas}}=M_{\mathrm{gas}} / M_{\mathrm{tot}}$ and characterize the efficiency with which AGNs input energy into the intracluster medium.

Long-term efforts to improve mass scaling relations focus on weak-lensing analyses, for example, from Euclid and LSST (Grandis et al. 2019). However, strong-lensing analyses will also contribute. Simulations have shown that joint stronglensing + weak-lensing analyses yield significantly smaller biases $(\sim 2 \%)$ and uncertainties $(\sim 20 \%)$ in virial mass estimates than either weak or strong lensing alone (Meneghetti et al. 2010). A joint strong-lensing plus weak-lensing analysis of 20 CLASH clusters based on HST and Subaru observations (Umetsu et al. 2016) shows that the $M_{2500}$ masses can be determined with a fractional total uncertainty of $25 \%$ per cluster at $\left\langle M_{2500}\right\rangle=3.6 \times 10^{14} M_{\odot} \quad\left(\right.$ or $\left.\left\langle M_{500}\right\rangle=10^{15} M_{\odot}\right)$, accounting for the dominant contributions from intrinsic profile variations (Gruen et al. 2015). Thus, a similar analysis of all 41 RELICS clusters would yield an overall mass calibration accuracy of about $4 \%$ from $r_{2500}$ out to the virial radii, providing a legacy mass profile database for critical tests for models of background cosmology and structure formation.

\subsection{Dark Matter Constraints}

Self-interacting dark matter (SIDM) particles with cross sections of $\sigma_{\mathrm{DM}} / m \sim 0.1-0.6 \mathrm{~cm}^{2} \mathrm{~g}^{-1}$ have been invoked to explain several observational inconsistencies with collisionless 
Table 6

RELICS HST Imaging with ACS and WFC3

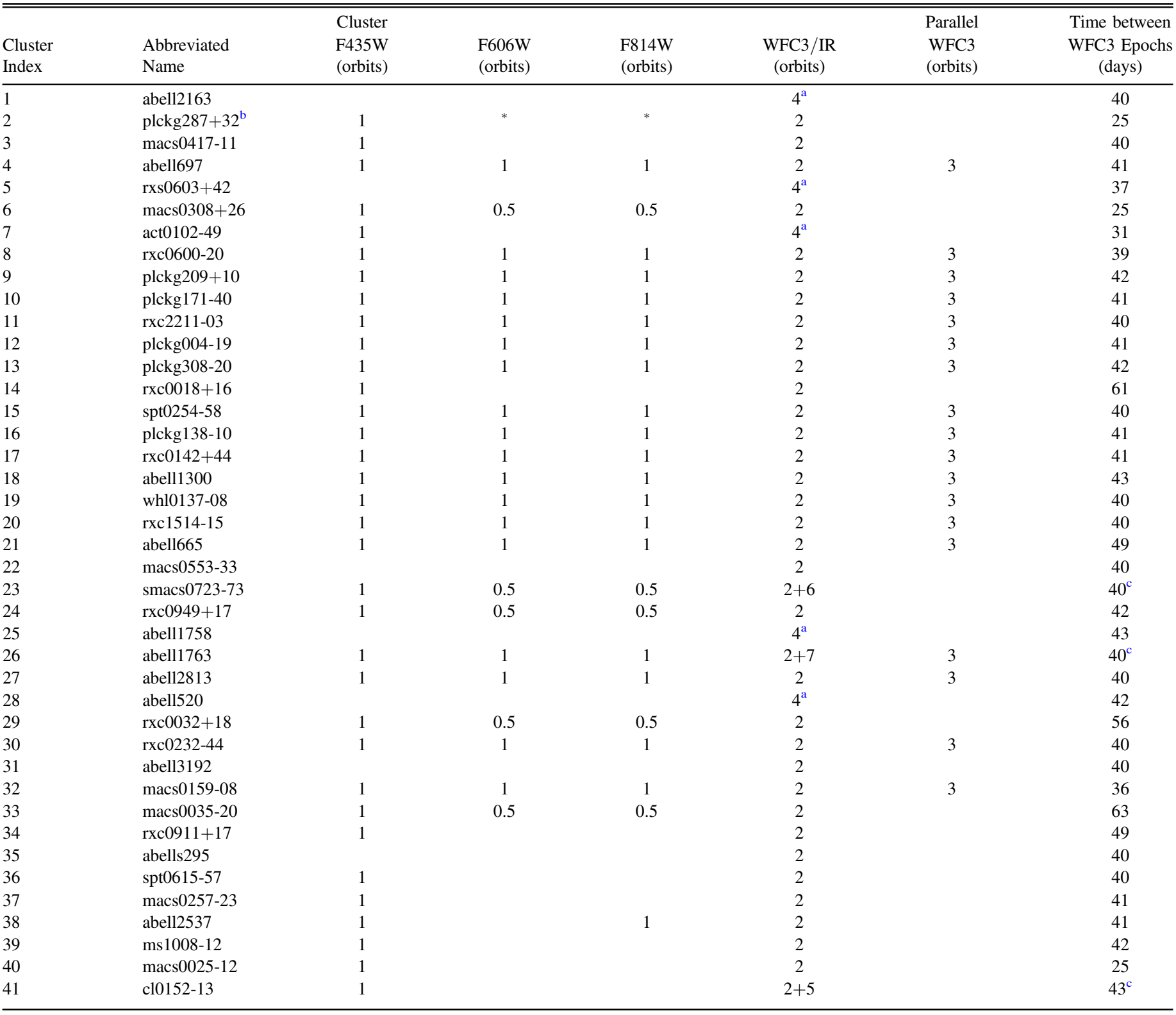

Notes.

a Observations were split between two pointings.

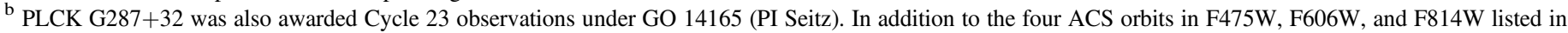

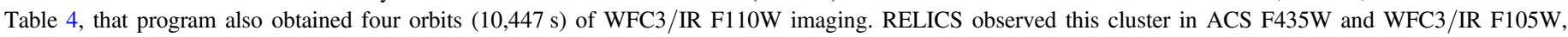
F125W, F140W, and F160W, relinquishing the two awarded orbits of F606W and F814W imaging that would have been duplicated.

c Indicates WFC3 orbits added for SN follow-up observations (Table 7). For those clusters, "Time between WFC3 Epochs" refers to the first two epochs.

cold dark matter (CDM) theory, including galaxy cluster density profiles, "missing" Milky Way satellites, and the "cusp-core" problem (see Robertson et al. 2019 and references therein). Baryonic processes can explain these "problems" with varying degrees of success. Merging galaxy clusters yield the most robust constraints on SIDM. As two clusters collide, galaxies pass straight through the collision, leaving the stripped cluster gas lagging behind. We expect dark matter to also pass straight through unless the particles have a significant self-interacting cross section. Joint HST lensing plus Chandra X-ray analysis of the Bullet Cluster (Clowe et al. 2006; Bradač et al. 2006) constrained $\sigma_{\mathrm{DM}} / m<1.25 \mathrm{~cm}^{2} \mathrm{~g}^{-1}$ (68\% confidence; Randall et al. 2008). More recently, Harvey et al. (2015) improved this constraint to $\sigma_{\mathrm{DM}} / m<0.47 \mathrm{~cm}^{2} \mathrm{~g}^{-1}$ (95\% confidence) by jointly analyzing $H S T+$ Chandra imaging of 30 cluster mergers (Bradač et al. 2008; Dawson et al. 2012). However, Wittman et al. (2018) subsequently analyzed more data (including more HST filters) on these same 30 clusters and claimed that the constraint should be more relaxed: $\sigma_{\mathrm{DM}} / m \lesssim 2 \mathrm{~cm}^{2} \mathrm{~g}^{-1}$.

RELICS includes seven cluster mergers analyzed by these papers plus another 17 confirmed plus nine possible mergers not in their sample. Of the confirmed mergers, all but one have 
Table 7

HST WFC3/IR and UVIS Follow-up Imaging of RELICS Supernovae

\begin{tabular}{|c|c|c|c|c|c|c|c|}
\hline Cluster & Supernova & Total Orbits & F105W & F125W & F140W & F160W & F350LP \\
\hline abell1763 & Nebra & 7 & 0.3 & 2.7 & & 3.7 & 0.3 \\
\hline clj0152-13 & Nimrud & 5 & & 2.5 & & 2.5 & \\
\hline smacs0723-73 & Yupana & 6 & 2 & 1 & 2 & 1 & \\
\hline
\end{tabular}

Note. The full list of RELICS SN discoveries is given in Table 18.

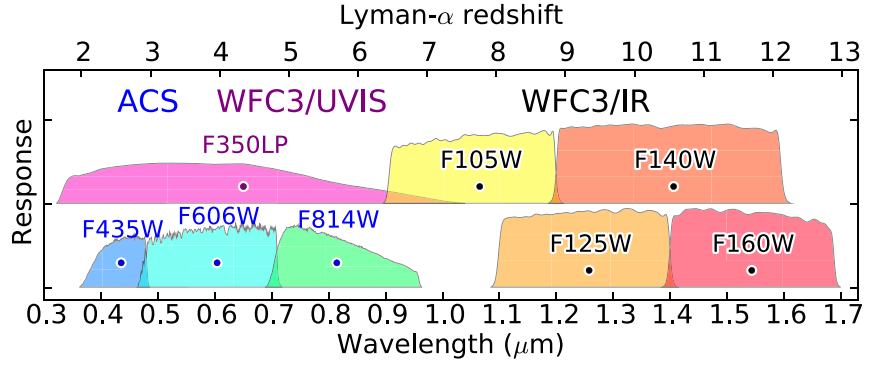

Figure 3. RELICS clusters were observed with the seven HST ACS and WFC3/IR filters shown here. RELICS parallels were observed with the one WFC3/UVIS and four WFC3/IR filters shown. Response curves are plotted vs. wavelength $(\lambda)$, with the corresponding $\operatorname{Ly} \alpha$ redshift $(z)$ given along the top axis $(\lambda=0.1216 \mu \mathrm{m}(1+z))$. F350LP, F105W, and F140W are offset vertically for clarity. Black dots mark the effective "pivot" wavelengths (Tokunaga \& Vacca 2005) of the filters.

existing Chandra imaging. Joint analyses of the lens models plus X-ray data of these massive cluster mergers (specifically, those observed post-merger) will help constrain the SIDM parameter space toward the astrophysically and theoretically interesting limit of $0.1 \mathrm{~cm}^{2} \mathrm{~g}^{-1}$.

\subsection{Supernovae}

Large HST programs have yielded many distant SNe. CLASH and CANDELS discovered both Type Ia SNe (SNe Ia) and core-collapse $\mathrm{SNe}(\mathrm{CCSNe})$ out to $z=2.5$. The observed SN Ia rates suggest that Type Ia progenitors are primarily double white dwarf systems (Graur et al. 2014) that do not explode quickly after formation (Rodney et al. 2014). High- $z$ CCSN rates from these programs reinforced measurements of the cosmic star formation rate history and put constraints on the initial mass function for CCSN progenitors (Strolger et al. 2015).

Lensed SNe Ia from CLASH and the Frontier Fields provided the first empirical tests verifying the accuracy of lens model magnification estimates (Nordin et al. 2014; Patel et al. 2014; Rodney et al. 2015b). The appearance of "SN Refsdal" (Kelly et al. 2015) was the first strongly lensed SN observed as multiple images with measurable time delays (Rodney et al. 2016b). These time delays can be used to test lens models (Kelly et al. 2016; Treu et al. 2016) or as a cosmological distance measurement (Grillo et al. 2018; Vega-Ferrero et al. 2018). Strong-lensing clusters have also revealed other faint transient phenomena, including extreme magnifications of individual stars or stellar eruptions in galaxies at $z=1.49$ (Kelly et al. 2018), $z=1.01$ (Rodney et al. 2018), and $z=0.94$ (Chen et al. 2019; Kaurov et al. 2019).

RELICS observed each cluster in two epochs separated by $\sim 40$ days to identify SNe and other transient phenomena. This cadence was designed to catch $z \sim 1-2 \mathrm{SNe}$ near peak brightness in epoch 2 . Our primary goal was to significantly
Table 8

Nominal Exposure Times and Expected Depths

\begin{tabular}{llcccc}
\hline \hline Camera & Filter & $\begin{array}{c}\lambda^{\mathrm{a}} \\
(\mu \mathrm{m})\end{array}$ & $\begin{array}{c}\text { Exp. }^{\mathrm{b}} \\
\text { Time }\end{array}$ & $\begin{array}{c}\text { Depth }^{\mathrm{c}} \\
(\mathrm{AB})\end{array}$ & $\begin{array}{c}\text { Sens. }^{\mathrm{d}} \\
(\mathrm{nJy})\end{array}$ \\
\hline HST ACS/WFC & F435W & 0.43 & $1952 \mathrm{~s}$ & 27.2 & 9 \\
$H S T$ ACS/WFC & F606W & 0.59 & $1991 \mathrm{~s}$ & 27.6 & 7 \\
$H S T$ ACS/WFC & F814W & 0.81 & $2123 \mathrm{~s}$ & 27.1 & 11 \\
$H S T$ WFC3/IR & F105W & 1.06 & $1361 \mathrm{~s}$ & 26.6 & 16 \\
$H S T$ WFC3/IR & F125W & 1.25 & $686 \mathrm{~s}$ & 26.0 & 29 \\
$H S T$ WFC3/IR & F140W & 1.39 & $686 \mathrm{~s}$ & 26.2 & 25 \\
$H S T$ WFC3/IR & F160W & 1.54 & $1861 \mathrm{~s}$ & 26.5 & 19 \\
SST IRAC & ch1 & 3.6 & $5 \mathrm{hr}$ & 25.0 & 76 \\
SST IRAC & ch2 & 4.5 & $5 \mathrm{hr}$ & 24.6 & 108 \\
\hline
\end{tabular}

Notes.

a Effective "pivot" wavelength (Tokunaga \& Vacca 2005).

b Total integration times for A1763; slightly longer integrations were obtained for most other clusters. Much deeper SST integrations (30 hr) were obtained for 10 RELICS clusters.

${ }^{c} 5 \sigma$ point-source $\mathrm{AB}$ magnitude limit. HST: within a 0 "! 4 diameter aperture assuming exposure time is split into four integrations. SST: assuming medium background with 180 integrations of $100 \mathrm{~s}$ each.

${ }^{\mathrm{d}} 1 \sigma$ point-source sensitivity (nJy) within the same apertures.

contribute to the numbers of known distant and lensed $\mathrm{SNe}$ discovered in previous surveys. Ultimately, RELICS discovered $11 \mathrm{SNe}$ (Section 6.3). The RELICS proposal included 20 orbits for follow-up imaging to confirm and obtain light curves for the more interesting candidates. RELICS observations will also provide a baseline for the longer-term (decade) monitoring required to detect higher-redshift $\mathrm{SNe}$ and other lensed transients, perhaps including individual Population III stars (Windhorst et al. 2018).

\section{Galaxy Clusters}

RELICS observed the 41 massive galaxy clusters at $0.182 \leqslant z \leqslant 0.972$ listed in Tables 2 and 3. None had existing $H S T$ infrared imaging (WFC3/IR or NICMOS) prior to RELICS. As shown in Figure 1, we selected 21 of the clusters based on exceptionally high mass estimates from Planck and the other 20 based on other factors revealing or suggesting exceptional lensing strength, including details noted in Table 3.

Planck Collaboration et al. (2016a) identified 1653 SZ (Sunyaev \& Zeldovich 1970) sources in their PSZ2 all-sky $\left(|b|>15^{\circ}\right)$ catalog ${ }^{36}$ including 1203 confirmed clusters, 1094 of which had measured redshifts and SZ mass estimates. A few hundred of these were newly discovered clusters, including some of the most massive known.

\footnotetext{
36 Planck PSZ2 catalogs are available and described at http://irsa.ipac.caltech. edu/data/Planck/release_2/catalogs/ and https://wiki.cosmos.esa.int/ planckpla2015/index.php/Catalogues
} 
Table 9

Nominal HST Exposures for a Cluster and Parallel Field with No Archival Imaging

\begin{tabular}{|c|c|c|c|c|c|}
\hline $\begin{array}{l}\text { Epoch/ } \\
\text { Orbit }\end{array}$ & $\begin{array}{l}\text { Dither }^{\mathrm{a}} \\
\text { Position }\end{array}$ & $\begin{array}{l}\text { Prime } \\
\text { Filter }\end{array}$ & $\begin{array}{l}\text { Exposure } \\
\text { Time (s) }\end{array}$ & $\begin{array}{l}\text { Parallel } \\
\text { Filter }\end{array}$ & $\begin{array}{c}\text { Exposure } \\
\text { Time (s) }\end{array}$ \\
\hline $1 / 1$ & A & $\mathrm{F} 435 \mathrm{~W}$ & 370 & F350LP & 454 \\
\hline $1 / 1$ & B & $\mathrm{F} 435 \mathrm{~W}$ & 667 & F350LP & 701 \\
\hline $1 / 1$ & $\mathrm{C}$ & F435W & 667 & F350LP & 701 \\
\hline $1 / 1$ & D & $\mathrm{F} 435 \mathrm{~W}$ & 371 & F350LP & 496 \\
\hline $1 / 2$ & A & F814W & 516 & F140W & 603 \\
\hline $1 / 2$ & B & F814W & 607 & F105W & 703 \\
\hline $1 / 2$ & $\mathrm{C}$ & F814W & 607 & F160W & 703 \\
\hline $1 / 2$ & D & F814W & 516 & F125W & 603 \\
\hline $1 / 3$ & W & F140W & 178 & & \\
\hline $1 / 3$ & W & F105W & 353 & & \\
\hline $1 / 3$ & $\mathrm{Y}$ & F105W & 353 & & \\
\hline $1 / 3$ & Y & F140W & 178 & & \\
\hline $1 / 3$ & Z & F125W & 178 & & \\
\hline $1 / 3$ & Z & F160W & 503 & & \\
\hline $1 / 3$ & $\mathrm{X}$ & F160W & 503 & & \\
\hline $1 / 3$ & $\mathrm{X}$ & $\mathrm{F} 125 \mathrm{~W}$ & 153 & & \\
\hline $2 / 4$ & A & F606W & 516 & F125W & 503 \\
\hline $2 / 4$ & B & F606W & 607 & F160W & 703 \\
\hline $2 / 4$ & $\mathrm{C}$ & F606W & 607 & F105W & 703 \\
\hline $2 / 4$ & D & F606W & 516 & F140W & 503 \\
\hline $2 / 5$ & Z & F140W & 178 & & \\
\hline $2 / 5$ & Z & F105W & 353 & & \\
\hline $2 / 5$ & $\mathrm{X}$ & F105W & 353 & & \\
\hline $2 / 5$ & $X$ & F140W & 203 & & \\
\hline $2 / 5$ & W & $\mathrm{F} 125 \mathrm{~W}$ & 178 & & \\
\hline $2 / 5$ & W & F160W & 503 & & \\
\hline $2 / 5$ & Y & F160W & 453 & & \\
\hline $2 / 5$ & $\mathrm{Y}$ & F125W & 230 & & \\
\hline
\end{tabular}

Notes. Each epoch begins with ACS on the prime cluster field and WFC3 in parallel and ends with WFC3/IR in prime. Epochs 1 and 2 are separated by about a month, or longer when possible.

${ }^{a}$ Each ACS dither position (ABCD) takes another step across the chip gap. The WFC3/IR dither positions consist of two close ( $\left.\sim 0^{\prime \prime} 8\right)$ pairs (WX and $\mathrm{YZ})$ separated by a larger distance $\left(\sim 66^{\prime \prime} 5\right)$ to cover the "death star."

Table 10

Spitzer IRAC Imaging Programs

\begin{tabular}{lccccc}
\hline \hline Program & PI & TAC & Hours $^{\mathrm{a}}$ & Depth $^{\mathrm{b}}$ & Clusters $^{\mathrm{c}}$ \\
\hline 12005 & Bradač & GO & 99.9 & 1.2 & 26 \\
12123 & Soifer & DDT & 290 & 5 & 34 \\
13165 & Bradač & DDT & 167 & 30 & 3 \\
13210 & Bradač & DDT & 55.5 & 30 & 1 \\
14017 & Bradač & GO & 333.2 & 30 & 6 \\
\hline
\end{tabular}

Notes.

${ }^{a}$ Total hours awarded, including overheads.

${ }^{\mathrm{b}}$ Resulting total hours observed in each IRAC filter $(\mathrm{ch} 1, \mathrm{ch} 2)$ for each cluster, including previous observations.

${ }^{c}$ Number of RELICS clusters observed. The first two programs observed all clusters as required to reach target depths. The final three programs observed 10 clusters to achieve $30 \mathrm{hr}$ depth for each.

We queried the HST observations of all the Planck clusters using the Mikulski Archive for Space Telescopes (MAST). ${ }^{37}$ Many had existing HST imaging prior to RELICS, but many more did not.

\footnotetext{
${ }^{37}$ http://archive.stsci.edu/hst/search.php
}

The 34 most massive clusters $\left(M_{500}>8.8 \times 10^{14} M_{\odot}\right)$ include some already well studied by $H S T$ and Spitzer, including $4 / 6$ Frontier Fields clusters ${ }^{38}$ and $5 / 25$ from CLASH (with three common to both surveys). But others were less well studied. Nine of the top 34 had yet to be observed by $H S T$ or Spitzer at all, and 12 more had yet to be observed by HST in the near-infrared with NICMOS or WFC3. These 21 clusters compose half of the RELICS sample; RELICS obtained the first HST infrared imaging of these clusters, and Spitzer imaging as needed.

The remaining 20 RELICS clusters are primarily known strong lenses based on existing HST optical imaging (or ground-based imaging in the case of RXC 0232-44; Kausch et al. 2010). Existing HST ACS imaging, where available, reduced our total orbit request. We also considered other factors in this selection, including X-ray mass estimates (Mantz et al. 2010; MCXC Piffaretti et al. 2011), weak-lensing mass estimates (Sereno 2015 compilation including Weighing the Giants; Applegate et al. 2014; von der Linden et al. 2014a; Umetsu et al. 2014; Hoekstra et al. 2015), SDSS clusters (Wen et al. 2012; Wong et al. 2013), and other SZ mass estimates from SPT (Bleem et al. 2015) and ACT (Hasselfield et al. 2013), as well as clusters nearly selected for the Frontier Fields. ${ }^{39}$

The 41 RELICS clusters generally bear the names of the surveys that discovered them: Abell (1958); Abell et al. (1989); the Einstein Observatory Extended Medium-Sensitivity Survey (MS; Gioia et al. 1990); the ROSAT X-ray All-Sky Galaxy Cluster Survey (RXC; Ebeling et al. 1998, 2000a); the ROSAT MAssive Cluster Survey (MACS and SMACS; Ebeling et al. 2001, 2007, 2010, 2013; Mann \& Ebeling 2012; Repp et al. 2016; Repp \& Ebeling 2018); an extended radio source discovered in ROSAT (RXS; van Weeren et al. 2012); the Wen, Han, \& Liu SDSS-III cluster catalog (WHL; Wen et al. 2012); the South Pole Telescope SZ survey (SPT; Bleem et al. 2015); the Atacama Cosmology Telescope SZ survey (ACT; Hasselfield et al. 2013); and the Planck all-sky SZ survey (Planck Collaboration et al. 2011, 2016a). CL J0152.7-1357 at $z=0.833$ was discovered by Ebeling et al. (2000b) in the Wide Angle ROSAT Pointed Survey (WARPS). Alternate cluster names include one also identified in the Rosat Bright Survey (RBS; Kausch et al. 2010) and another in Clusters in the Zone of Avoidance (CIZA; Kocevski et al. 2007).

The Abell clusters are numbered sequentially based on the original catalog. Numbers in other cluster names codify coordinates, often in J2000 R.A. HH:MM and decl. DD:MM. Planck cluster names, instead, give Galactic coordinates in longitude and latitude. Table 3 lists names and any alternate names for each RELICS target. Their positions on the sky are shown in Figure 2.

\section{Observations}

All data from our large $H S T$ and Spitzer observing programs had no proprietary period. We describe each of these programs

\footnotetext{
38 The Frontier Fields cluster $\mathrm{A} 370$ has a lower Planck mass $M_{500}=7.7 \times 10^{14} M_{\odot}$ (though weak-lensing analysis yields a far greater $M_{500}=1.9 \times 10^{15} M_{\odot}$; Umetsu et al. 2011). MACS J0416.1-2403 is PSZ2 G221.06-44.05, but the association was not initially made; without the redshift, no mass estimate was possible in the PSZ2 catalog. However, the relatively low Planck signal-to-noise ratio of 5.2 suggests a lower mass.

39 https://outerspace.stsci.edu/display/HPR/Frontier+Fields+Cluster+ Candidate + Selection
} 
Table 11

Large Surveys Including RELICS Clusters Motivated in Part by RELICS

\begin{tabular}{|c|c|c|c|c|c|c|}
\hline Name & PI & Observatory & Instrument & Time & Wavelength & RELICS/Total Clusters \\
\hline RELICS $^{\mathrm{a}}$ & Coe & $H S T$ & ACS, WFC3 & $115 \mathrm{hr}$ & $0.4-1.7 \mu \mathrm{m}$ & 41 \\
\hline S-RELICS ${ }^{\mathrm{b}}$ & Bradač; Soifer & Spitzer & IRAC & $945 \mathrm{hr}$ & $3-5 \mu \mathrm{m}$ & $34^{\mathrm{c}}$ \\
\hline GAME & Mercurio & VST & OmegaCAM & $300 \mathrm{hr}$ & ugri & $9 / 12$ \\
\hline GCAV $^{\mathrm{d}}$ & Nonino & VISTA & VIRCAM & $540 \mathrm{hr}$ & YJKs & $13 / 20$ \\
\hline Witnessing ${ }^{\mathrm{e}}$ & Arnaud \& Ettori & XMM-Newton & EPIC & $833 \mathrm{hr}$ & $0.15-15 \mathrm{keV}$ & $18 / 118$ \\
\hline ALCS & Kohno & ALMA & $12 \mathrm{~m}$ array & $95 \mathrm{hr}$ & $1.1 \mathrm{~mm}$ & $16 / 33$ \\
\hline Probing $^{\mathrm{f}}$ & van Weeren & VLA & $25 \mathrm{~m}$ array & $85 \mathrm{hr}$ & $2-4 \mathrm{GHz}$ & $34^{\mathrm{g}}$ \\
\hline
\end{tabular}

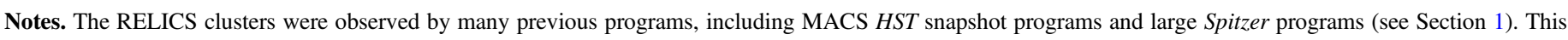
table does not include those, instead summarizing the more recent programs inspired in part by the RELICS program.

a https://relics.stsci.edu

${ }^{\mathrm{b}}$ https://irsa.ipac.caltech.edu/data/SPITZER/SRELICS/overview.html

c The remaining seven RELICS clusters already had archival $5 \mathrm{hr}$ depth, and none of them were targeted for $30 \mathrm{hr}$ depth.

${ }^{d}$ http://archive.eso.org/cms/eso-archive-news/first-data-release-from-the-galaxy-clusters-at-vircam-gcav-eso-vista-public-survey.html

e Witnessing the culmination of structure formation in the universe.

${ }^{\mathrm{f}}$ Probing cosmic star formation with the JVLA Lensing Cluster Survey.

g All RELICS clusters accessible to VLA.

in turn, followed by other subsequent large observing programs of RELICS clusters.

\subsection{HST Imaging}

Of the 41 RELICS clusters, 28 had been observed previously by HST with ACS and/or WFC3/UVIS (Tables 4 and 5). Our 188-orbit HST Treasury Program (Cycle 23; GO 14096; PI Coe; Deputy PI Bradley) obtained additional observations with ACS and WFC3/IR (Table 6). Five clusters required two WFC3/IR pointings for a total of 46 new WFC3/IR images of strongly lensed fields. For each field, we observed two orbits of WFC3/IR split among four filters: F105W, F125W, F140W, and F160W (Figure 3). And for each cluster, we observed three orbits of ACS split among F435W, F606W, and F814W, minus any archival imaging. For the 18 RELICS clusters without any existing ACS imaging, we observed the full three orbits of ACS and, in parallel, three orbits of WFC3 on a blank field: one orbit of WFC3/UVIS F350LP and two orbits of WFC3/IR F105W, F125W, F140W, and F160W. The parallels use the same filters as in BoRG[z9-10] (Calvi et al. 2016; Morishita et al. 2018), adding area to our high- $z$ search.

For each cluster, we split the observations into two epochs separated by $25-63$ days (typically $\sim 40$; see Table 6 ). This enabled searches for SNe and other transients. Twenty orbits of RELICS were allocated to follow up such Targets of Opportunity (ToO). We executed these orbits on three of the nine SNe discovered by RELICS (Table 7).

Total integration times for each HST orbit vary with the time available between Earth occultations and the time to acquire or reacquire guide stars. Table 8 gives the total integration times in each filter for A1763, as a representative example. For most other clusters, the integration times were slightly longer (by up to $15 \%$ ). Each integration was split into four exposures at different dither positions (pointings). We dithered across the ACS chip gap and WFC3/IR "death star" to fill these gaps in the data.

Table 9 gives the detailed breakdown of epochs, orbits, and exposure times at each of the four dither positions in each filter for a nominal target with no archival imaging. In the parallel fields, note that the WFC3/UVIS F350LP imaging consists of four exposures at four dither positions, but the parallel WFC3/ IR imaging consists of only two exposures in each filter (one per epoch).

\subsection{Spitzer Imaging}

Altogether, RELICS has been awarded $945 \mathrm{hr}$ of Spitzer observing time (Table 10). For each cluster, we obtained IRAC imaging as needed in the two warm mission filters, ch1 $(3.6 \mu \mathrm{m})$ and $\operatorname{ch} 2(4.5 \mu \mathrm{m})$. Combined, these filters span approximately $3-5 \mu \mathrm{m}$. About $100 \mathrm{hr}$ of archival IRAC imaging were also available for 18 of the clusters in these two filters, most notably from GO 60034 (PI Egami). Below we summarize the complete Spitzer RELICS data sets, which will be detailed further by V. Strait et al. (2019, in preparation).

Our initial $100 \mathrm{hr}$ SRELICS (Spitzer RELICS) program (Cycle 12; GO 12005; PI Bradač) was supplemented by a 290 hr Director's Discretionary Time program (DDT 12123; PI Soifer). Combined, these programs observed all RELICS clusters (as needed) to achieve a total of $5 \mathrm{hr}$ of integration time (combining new and archival imaging) in each of the two IRAC filters. The five clusters requiring two WFC3/IR pointings also required two IRAC pointings.

Subsequently, based on our analyses of the HST and Spitzer images, we were awarded three more proposals (PI Bradač) to obtain deeper IRAC imaging (30 hr/band), requiring an additional $556 \mathrm{hr}$ on the 10 clusters yielding the most high- $z$ candidates at $z \sim 6-10$ (Salmon et al. 2017, 2018). DDT 13165 observed PLCK G287.0+32.9, PLCK G004.5-19.5, and A1763. DDT 13210 observed SPT-CL J0615-5746 and the $z \sim 10$ arc discovered by Salmon et al. (2018). And GO 14017 observed CL J0152.7-1357, ACT-CL J0102-49151, PLCK G308.3-20.2, RXS J060313.4+421, MS 1008.1-1124, and SMACS J0723.3-7327.

\subsection{Other Large Surveys of RELICS Clusters}

In addition to the large $H S T$ and SST observing programs, RELICS has also motivated large surveys with other telescopes, including VST, VISTA, XMM-Newton, ALMA, and VLA (see Table 11). The VLA survey exclusively observes RELICS clusters, while the other listed programs have most or 

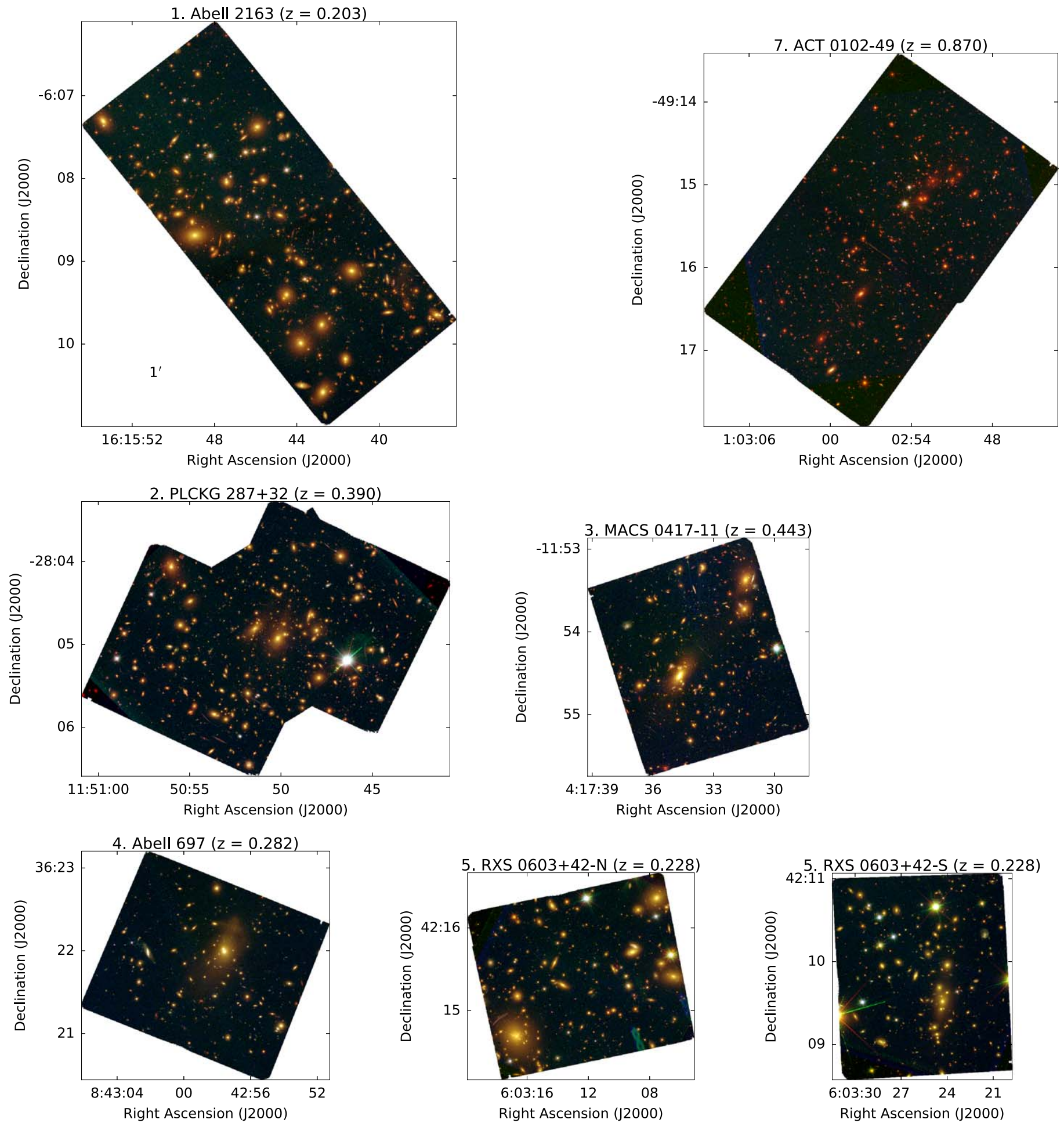

Figure 4. HST ACS + WFC3/IR observations of RELICS clusters within the WFC3/IR footprints. ACS imaging extends to wider areas not shown. All images are shown to the same scale. North is up; east is left. Color images produced using Trilogy (Coe et al. 2012): blue $=$ F435W; green $=$ F606W + F814W; red $=$ F105W $+\mathrm{F} 125 \mathrm{~W}+\mathrm{F} 140 \mathrm{~W}+\mathrm{F} 160 \mathrm{~W}$.

just some clusters in common with RELICS. We have also carried out smaller follow-up programs with many other observatories, including Keck MOSFIRE and DEIMOS, VLT MUSE and X-SHOOTER, Gemini GMOS, Subaru SuprimeCam and HSC, Magellan MegaCam and LDSS3, MMT Hectospec, and GMRT. Spectra from Magellan LDSS3 have already been used in several papers (Cerny et al. 2018; PaternoMahler et al. 2018; Mainali et al. 2019). Also note that while we are advertising these newer surveys here, we also emphasize that many RELICS clusters have been observed previously by large programs carried out with HST, Spitzer, Chandra, XMM-Newton, and ground-based observatories. 

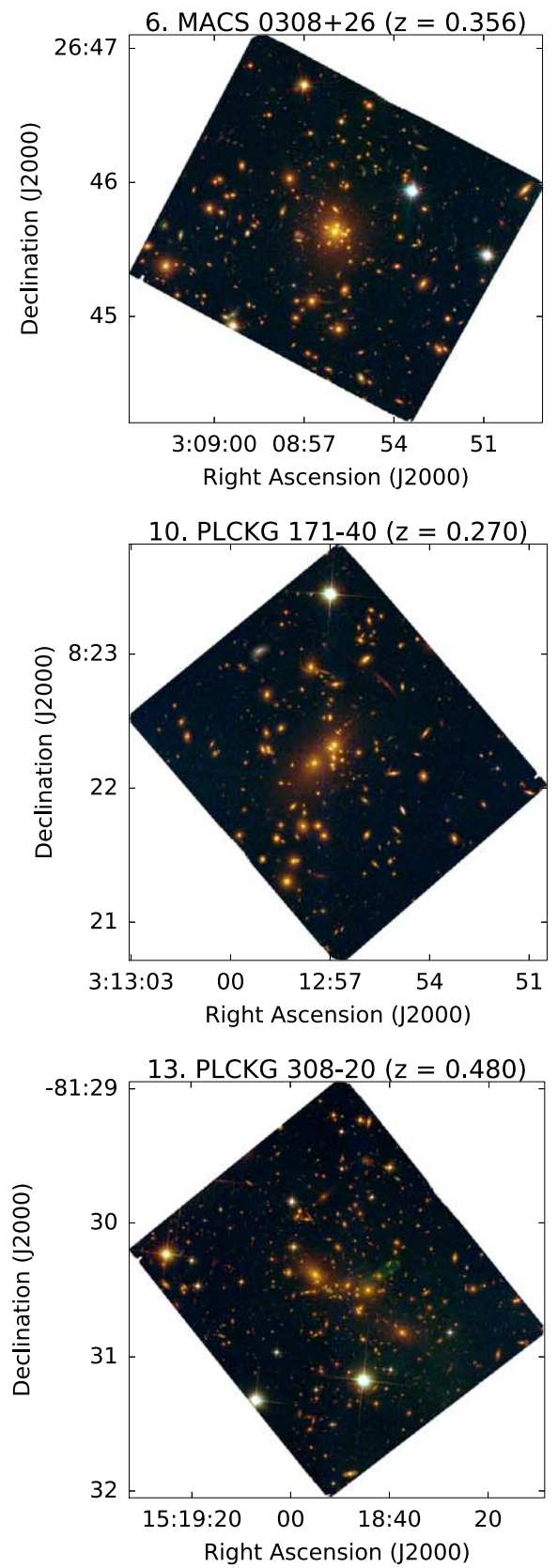

\section{Image Reductions and Catalogs}

We have made our HST reduced images and catalogs publicly available via MAST ${ }^{40}$ at doi:10.17909/T9SP45 and our Spitzer reduced images available via IRSA. ${ }^{41}$ Below we describe the procedures used to generate these data products.

\subsection{HST Image Reductions}

We reduced all HST images obtained by RELICS and all archival HST ACS and WFC3/IR images that overlap with the RELICS WFC3/IR images. Our HST image reduction procedure is similar to that performed on the Frontier Fields HST images

\footnotetext{
${ }^{40}$ https://archive.stsci.edu/prepds/relics/

${ }^{41}$ https://irsa.ipac.caltech.edu/data/SPITZER/SRELICS/
}
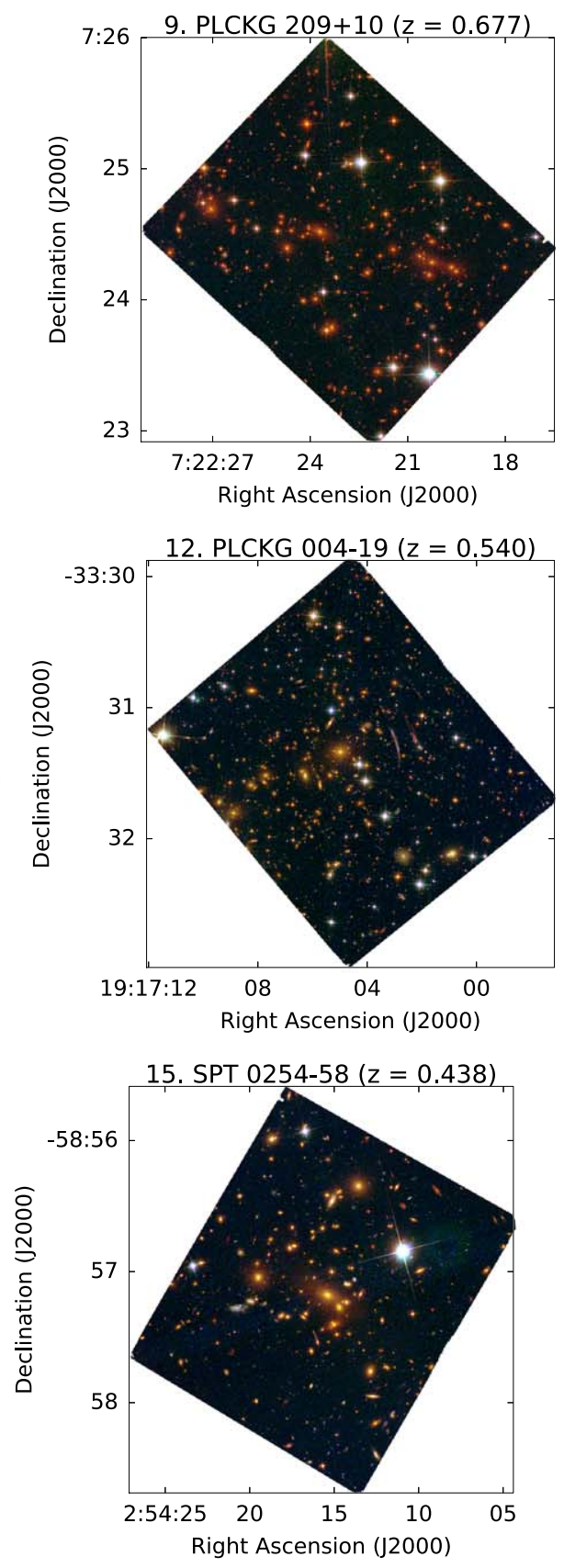

Figure 4. (Continued.)
(Lotz et al. 2017). Key differences are that we do not produce "self-calibrated" ACS images (due to insufficient numbers of exposures), nor do we correct for time-variable sky emission due to helium line emission at $1.083 \mu \mathrm{m}$, which occasionally affects WFC3/IR F105W images (Brammer et al. 2014).

We correct all HST images for bias, dark current, and flat fields with up-to-date reference files (as noted in the FITS headers of our final reduced images). We manually identified and masked any satellite trails. ACS images were corrected for charge transfer efficiency (CTE) and bias striping. The multiple ACS exposures were used to automatically identify and reject cosmic rays. Each WFC3/IR MULTIACCUM exposure consists of multiple samples, enabling "up-the-ramp" cosmic-ray rejection. We also masked bad pixels using updated identifications in WFC3/IR images from GO 14114 (G. Brammer 2019, private communication). 

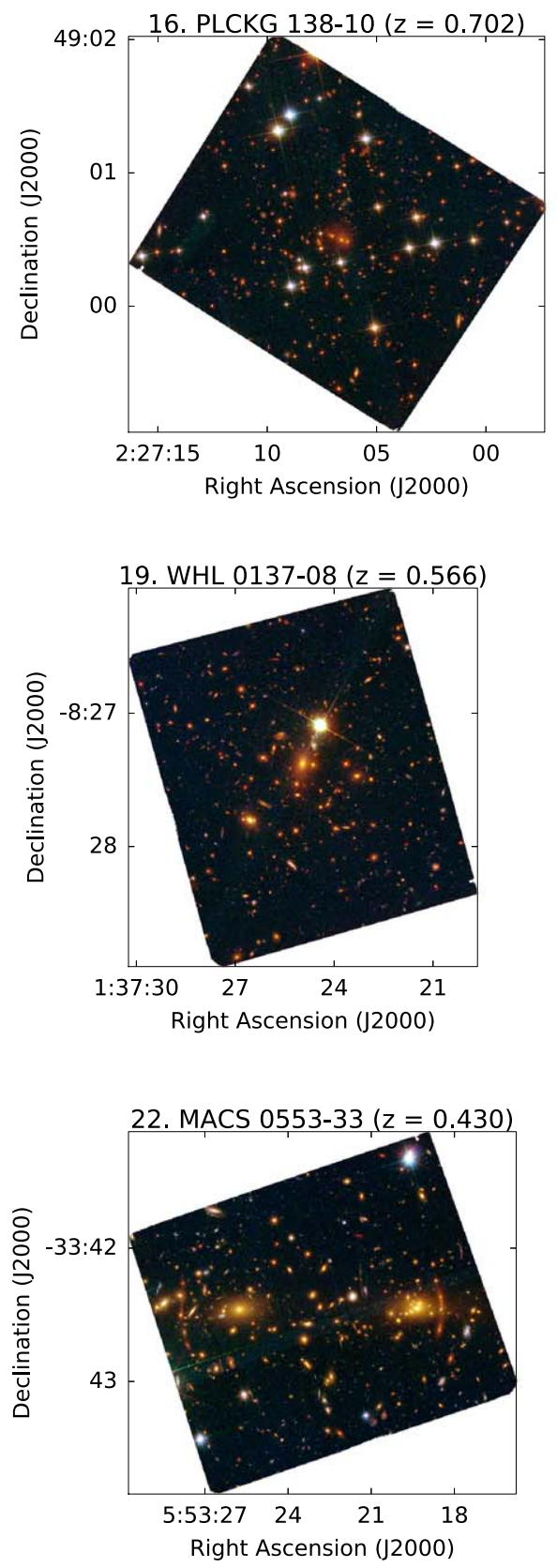

We produced inverse variance maps (IVMs) quantifying the uncertainty in each pixel before accounting for correlated pixel noise (an additional 10\%-15\%) and Poisson source noise. These IVMs were used as weights to drizzle-combine the images obtained in each filter.

For each cluster or parallel field, we aligned all processed HST images to two common grids with $0 . " 06$ and 0 ". 03 resolution. These are meant to adequately sample the point-spread functions (PSFs) in the WFC3/IR images (FWHM 0."18) and ACS images (FWHM $\sim 0$ ". 09), respectively. We used procedures from DrizzlePac, specifically AstroDrizzle (Gonzaga et al. 2012), and as outlined in Koekemoer et al. $(2002,2011)$ and Lotz et al. (2017). We set the drizzle parameter pixfrac $=0.8$, as used in programs such as CANDELS (see discussion in Section 5.8.2 of Koekemoer et al. 2011).
We corrected the absolute astrometry of our images using the Wide-field Infrared Survey Explorer (WISE) point-source catalog (Wright et al. 2010).

Finally, we produced automatically scaled color images using Trilogy (Coe et al. 2012). Figure 4 show HST ACS + WFC3 color images of all 46 WFC3/IR cluster fields observed by RELICS. The ACS color images extend to wider areas, which are not shown here but are available on MAST. Also on MAST are color images of the 18 RELICS parallel fields observed with WFC3 UVIS and IR.

\subsection{HST Detection and Photometry Catalogs}

Based on the 0." 06 resolution HST images, we produced source catalogs using techniques similar to those employed for the public 

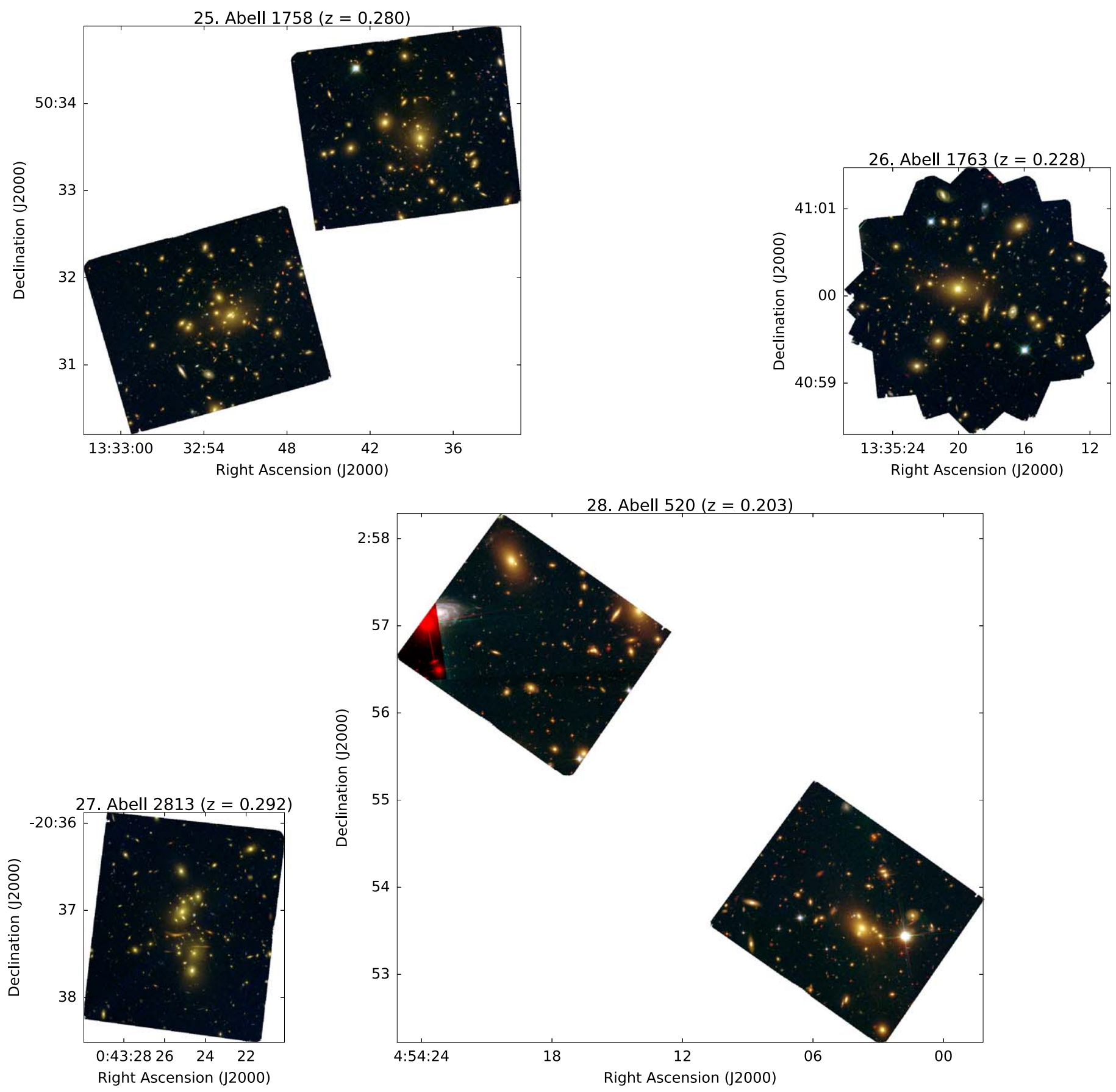

Figure 4. (Continued.)

CLASH catalogs (Postman et al. 2012; Coe et al. 2013) and the Frontier Fields analysis presented in Coe et al. 2015.

We ran SExtractor (Bertin \& Arnouts 1996) version 2.8.6 in dual-image mode to detect objects in each field and define their isophotal apertures for photometry to be measured in each filter image. For each field, we produce two source catalogs:

1. acs-wfC3 (or acs-wfc3ir): based on detections in a weighted stack of all HST images (ACS, WFC3/UVIS, and WFC3/IR), optimized to detect most objects.

2. wfC3ir: based on detections in a weighted stack of WFC3/IR images only using a finer background grid and more aggressive deblending, optimized to detect smaller high-redshift galaxies.

The stacked images are weighted sums; the weights are the IVMs produced by the drizzling software (Section 5.1). For SExtractor input, we also produce rms maps equal to $1 /$ sqrt (weight).

Table 12 lists the SExtractor parameters used for the acswfc3 and wfc3ir catalogs. The latter is geared toward detections of small high-redshift galaxies, so we use a smaller background grid and more aggressive deblending to detect small objects near brighter ones. The acs-wfc3 catalogs 

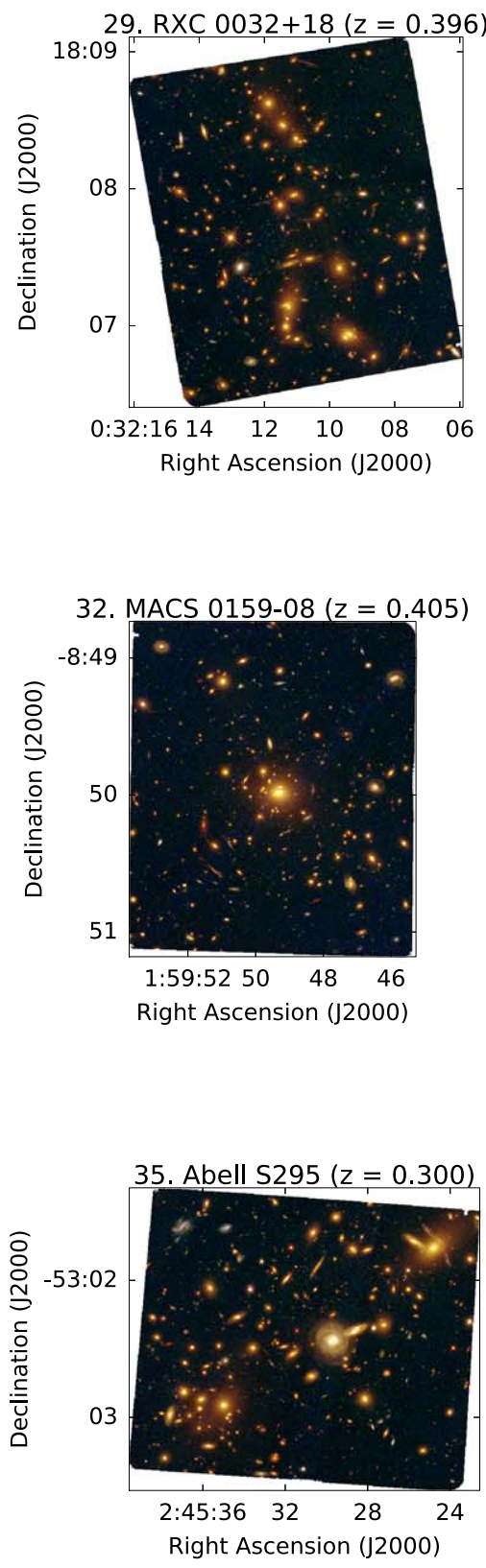
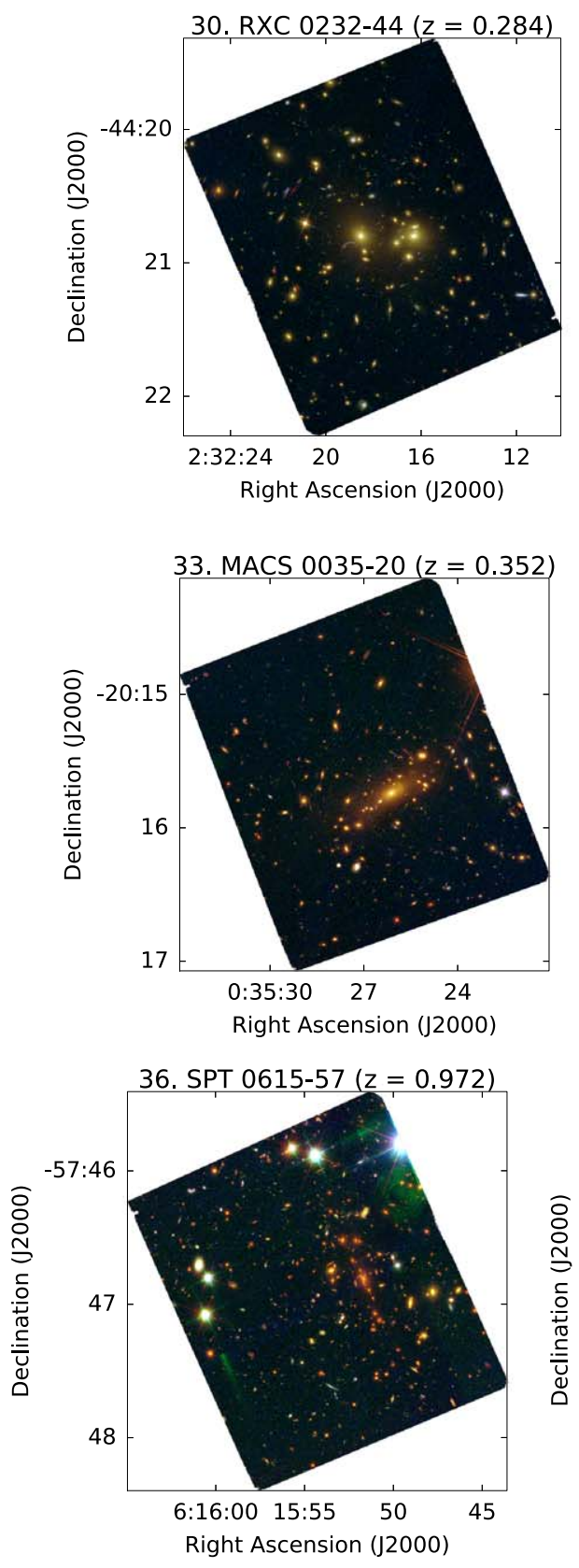

Figure 4. (Continued.)
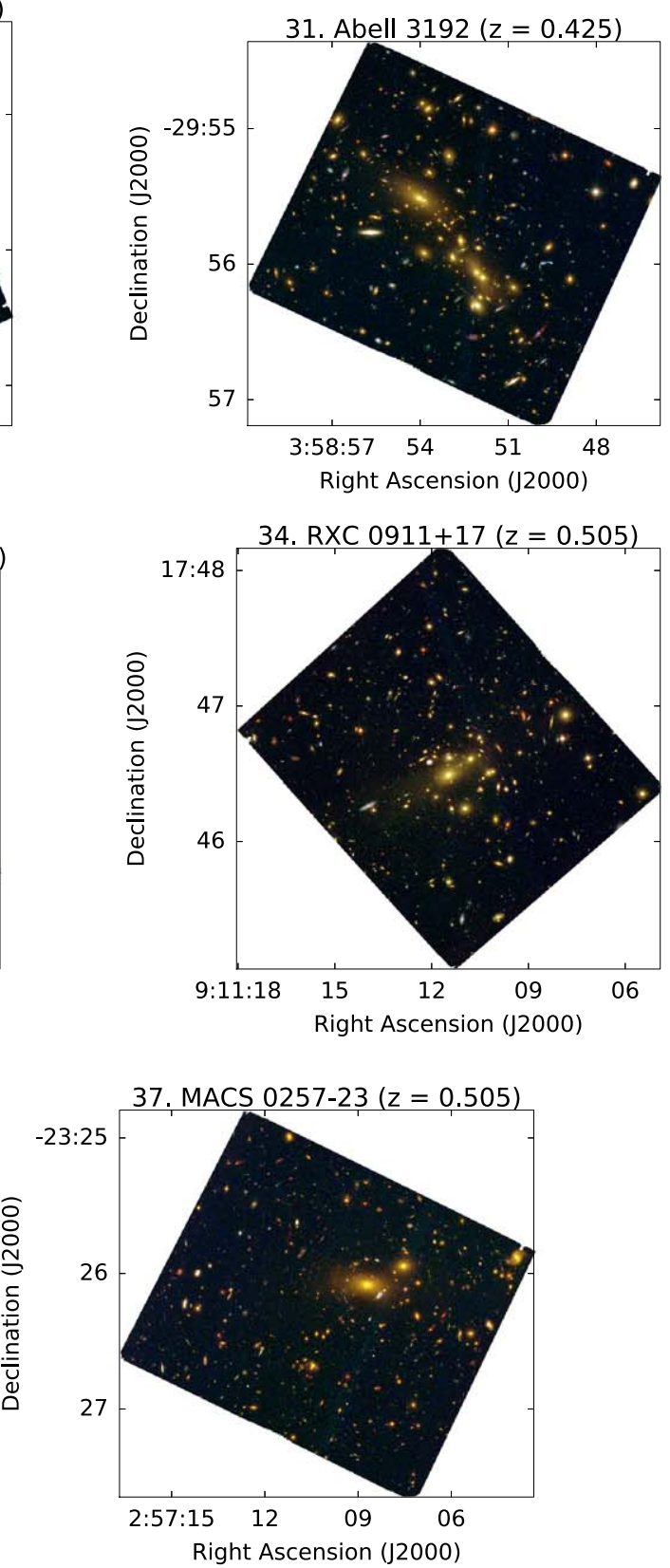

cover the full (larger) ACS field of view and aim to detect whole objects, breaking them apart less often.

For detections in either catalog, 9 contiguous pixels are required at the level of the observed background rms or higher. The acs-wfc3 (wfc3ir) background is calculated in $5 \times 5$ $(3 \times 3)$ grids of cells with $128 \times 128(64 \times 64)$ pixels in each cell. We set the deblending of adjacent objects to 128 (64) levels of 0.0015 (0.0001) minimum contrast.

Each object's detection defines an isophotal aperture, which SExtractor uses in dual-image mode to measure isophotal photometry in every filter in the aligned images. Just outside this aperture, we have SExtractor use a 24-pixel-wide rectangular annulus around each object to estimate and subtract the local background in each filter. We do not perform aperture corrections, as the PSF FWHMs only vary between $\sim 0$ !" 07 and
0 ". 15 and we use relatively large isophotal apertures (see discussion in Postman et al. 2012, Section 5.1).

Finally, we correct all photometry for Galactic extinction using the IR dust emission maps of Schlafly \& Finkbeiner (2011). ${ }^{42}$ Table 2 gives the extinction $E(B-V)$ for each cluster from those maps. These are multiplied by coefficients $A_{\lambda}$ (Table 13) for each filter to determine the extinction in magnitudes.

Table 14 summarizes the output in our HST catalogs available on MAST. Samples are provided from our A697 IR-detection catalog for object detection and shape measurement (Table 15), photometry (Table 16), and Bayesian photometric redshifts (Table 17), which are discussed below.

\footnotetext{
$\overline{42}$ Dust extinctions extracted using http://irsa.ipac.caltech.edu/applications/ DUST/docs/dustProgramInterface.html.
} 

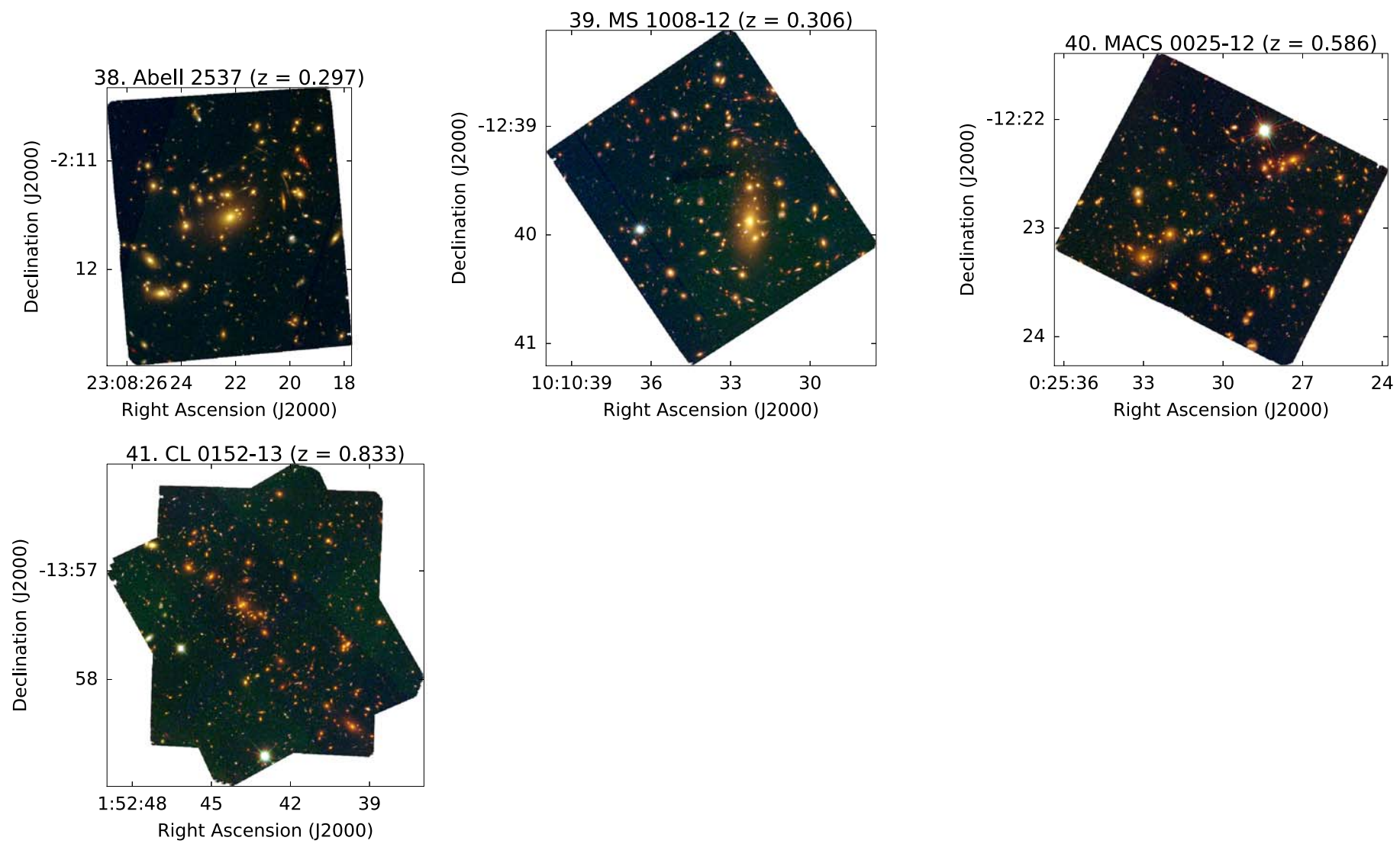

Figure 4. (Continued.)

Table 12

SExtractor Parameters Used in the RELICS HST Source Catalogs

\begin{tabular}{lccl}
\hline \hline SExtractor Parameter & acs-wfc3 & wfc3ir & Description \\
\hline DETECT_MINAREA & 9 & 9 & Contiguous pixels required above detection threshold \\
DETECT_THRESH & 1 & 1 & Detection threshold ( $\sigma$ above background rms) \\
BACK_SIZE & 128 & 64 & Background cell size \\
BACK_FILTERSIZE & 5 & 3 & Background grid size \\
DEBLEND_NTHRESH & 32 & 64 & Number of threshold levels \\
DEBLEND_MINCONT & 0.0015 & 0.0001 & Minimum contrast ratio \\
BACKPHOTO_TYPE & LOCAL & LOCAL & Method for measuring background \\
BACKPHOTO_THICK & 24 & 24 & Width of rectangular annulus around each object \\
\hline
\end{tabular}

As a caution to users, we note that the source catalogs include image artifacts such as diffraction spikes and objects poorly segmented by SExtractor. Additionally, photometry is complicated in crowded fields, especially as brighter cluster members contaminate the light from fainter, more distant objects.

\subsection{Photometric Redshifts}

Based on the HST photometry, we measured photometric redshifts using Bayesian Photometric Redshifts (BPZ; Benítez 2000; Coe et al. 2006) and Easy and Accurate $z_{\text {phot }}$ from Yale (EAZY; Brammer et al. 2008), which were two of the top performing methods in controlled tests (Hildebrandt et al. 2010).

For BPZ, we used 11 spectral models shown in Figure 5 and described in Coe et al. (2013), Benítez et al. (2014), and Rafelski et al. (2015). Briefly, the model spectral energy
Table 13

Galactic Reddening Extinction Correction Coefficients $A_{\lambda}$ for Each Filter

\begin{tabular}{llc}
\hline \hline Camera & Filter & Coefficient \\
\hline WFC3/UVIS & F390W & 4.514 \\
ACS & F435W & 4.117 \\
ACS & F475W & 3.747 \\
ACS & F555W & 3.242 \\
ACS, WFC3/UVIS & F606W & 2.929 \\
ACS & F625W & 2.671 \\
ACS & F775W & 2.018 \\
ACS & F814W & 1.847 \\
ACS & F850LP & 1.473 \\
WFC3/IR & F105W & 1.015 \\
WFC3/IR & F110W & 0.876 \\
WFC3/IR & F125W & 0.757 \\
WFC3/IR & F140W & 0.609 \\
WFC3/IR & F160W & 0.470 \\
\hline
\end{tabular}


Table 14

HST Source Catalog Content

\begin{tabular}{|c|c|c|}
\hline Column & Parameter & Description \\
\hline 1 & id & Object ID number \\
\hline 2 & R.A. & Right ascension in decimal degrees (J2000) \\
\hline 3 & Decl. & Declination in decimal degrees (J2000) \\
\hline 4 & $x$ & $x$ pixel coordinate \\
\hline 5 & $y$ & $y$ pixel coordinate \\
\hline 6 & fwhm & Full width at half maximum $(\operatorname{arcsec})$ \\
\hline 7 & area & Isophotal aperture area (pixels) \\
\hline 8 & stel & SExtractor "stellarity" $(1=$ star; $0=$ galaxy $)$ \\
\hline 9 & ell & Ellipticity $=1-B / A$ \\
\hline 10 & theta & Position angle (CCW wrt $x$ axis; degrees) \\
\hline 11 & nf5sig & Number of filters with a $5 \sigma$ detection \\
\hline 12 & nfobs & $\begin{array}{l}\text { Number of filters observed for this object (in } \\
\text { the field of view and without bad pixels) }\end{array}$ \\
\hline 13 & f435w_mag & $\begin{array}{l}\text { F435W isophotal magnitude }(99=\text { non- } \\
\text { detection; }-99=\text { unobserved })\end{array}$ \\
\hline 14 & f435w_magerr & $\begin{array}{l}\text { F435W isophotal magnitude uncertainty (or } \\
1 \sigma \text { upper limit for nondetection) }\end{array}$ \\
\hline 15 & f435w_flux & F435W isophotal flux $\left(e^{-} \mathrm{s}^{-1}\right)$ \\
\hline 16 & f435w_fluxerr & F435W isophotal flux uncertainty $\left(e^{-} \mathrm{s}^{-1}\right)$ \\
\hline 17 & f435w_fluxnJy & F435W isophotal flux (nJy) \\
\hline 18 & f435w_fluxnJyerr & F435W isophotal flux uncertainty (nJy) \\
\hline 19 & f435w_sig & F435W detection significance \\
\hline$\cdots$ & $\cdots$ & (photometry in other filters) \\
\hline $62^{\mathrm{a}}$ & bright_mag & Brightest magnitude in any filter \\
\hline 63 & bright_magerr & Brightest magnitude uncertainty \\
\hline 64 & $\mathrm{zb}$ & $\begin{array}{l}\text { BPZ most likely Bayesian photometric } \\
\text { redshift }\end{array}$ \\
\hline 65 & zbmin & BPZ lower limit (95\% confidence) \\
\hline 66 & zbmax & BPZ upper limit (95\% confidence) \\
\hline 67 & tb & $\begin{array}{l}\text { BPZ most likely spectral type (1-5 elliptical; } \\
6-7 \text { spiral; } 8-11 \text { starburst) }\end{array}$ \\
\hline 68 & odds & $P(z)$ contained within $\mathrm{zb} \pm 0.04(1+z)$ \\
\hline 69 & chisq & $\begin{array}{l}\chi^{2} \text { poorness of BPZ fit: observed versus - } \\
\text { model fluxes }\end{array}$ \\
\hline 70 & chisq2 & $\begin{array}{l}\text { Modified } \chi^{2} \text { : model fluxes allowed uncer- } \\
\text { tainties (Coe et al. 2006) }\end{array}$ \\
\hline 71 & M0 & $\begin{array}{l}\text { Magnitude used as BPZ prior: F775W or } \\
\text { closest available filter }\end{array}$ \\
\hline 72 & $\mathrm{zml}$ & $\begin{array}{l}\text { Maximum likelihood (flat prior) most likely } \\
\text { redshift }\end{array}$ \\
\hline 73 & tml & $\begin{array}{l}\text { Maximum likelihood (flat prior) most likely } \\
\text { spectral type }\end{array}$ \\
\hline
\end{tabular}

Note.

${ }^{\text {a }}$ Column numbers will vary depending on the number of HST filters observed in each field.

distributions (SEDs) are originally from PEGASE (Fioc \& Rocca-Volmerange 1997) but recalibrated, based on observed photometry and spectroscopic redshifts from FIREWORKS (Wuyts et al. 2008). The templates were selected to encompass ranges of metallicities, extinctions, and star formation histories observed for the vast majority of real galaxies. We allowed BPZ to interpolate nine templates between each pair of adjacent templates, yielding 101 templates altogether. BPZ fit the photometry to a grid of these 101 templates and 1300 redshifts linearly spaced from $z=0.001$ to $z=13$. BPZ tempers those $\chi^{2}$ results with a Bayesian prior $P(z, T \mid m)$, which gives the likelihood of a redshift $z$ and template type $T$ given an observed magnitude $m$ in $\mathrm{F} 814 \mathrm{~W}$. We used the original BPZ prior derived from the HDFN (Benítez 2000) and plotted in Figure 6 for $m=24,26$, and $\geqslant 32$, the latter including $\mathrm{F} 814 \mathrm{~W}$ nondetections. We do not attempt to correct magnitudes for lensing magnifications in our initial catalogs because those estimates are not available from the start. We note that our prior's dependence on magnitude is often gradual, but magnification should be accounted for in a more accurate $P$ (z) estimate.

We used EAZY (Brammer et al. 2008) to obtain a second independent set of photometric redshift estimates. EAZY uses a different template set and allows interpolation between any pair of templates. The nine templates used here include seven from PEGASE, one very dusty and quiescent galaxy from Maraston (2005), and one extreme emission line galaxy (EELG) from Erb et al. (2010). We used a flat prior with EAZY, as we found that the default prior was systematically biased against highredshift galaxies, strongly preferring lower-redshift EELGs with worse fits to the photometry (see discussion in Salmon et al. 2017).

Salmon et al. (2017) present a comparison of the templates and results from BPZ and EAZY for RELICS high- $z$ candidates.

\subsection{Spitzer Image Reductions}

We reduced the Spitzer images using MOPEX (Makovoz \& Khan 2005) and generated catalogs using T-PHOT (Merlin et al. 2015). The reduced images are available via IRSA. Currently these include data from programs 12005 and 12123 (totaling $5 \mathrm{hr}$ of depth per filter). Deeper data from the additional three Spitzer programs (Table 10) will be included in future releases of reduced images. More details will be presented by V. Strait et al. (2019, in preparation). Due to the broader Spitzer PSF, extra care is required in obtaining aperture-matched HST + Spitzer photometry. Less careful Spitzer photometry can result in less accurate photometric redshifts (Hildebrandt et al. 2010). We did not use the Spitzer photometry in our initial photometric redshift catalog release. We did use the Spitzer photometry to vet our $z \sim 10$ candidates, and we will use it to study the properties of all our high-redshift candidates.

\section{Results}

To date, RELICS has delivered the following science results on high-redshift galaxies (Section 2.1), strong-lens modeling (Section 2.2), and $\mathrm{SNe}$ (Section 2.5). Reduced images, catalogs, and lens models are available via MAST and IRSA. Constraints on cluster masses (Section 2.3) and the dark matter particle cross section (Section 2.4) require weak-lensing data and analyses on a longer timescale.

\subsection{High-redshift Candidates}

RELICS yielded over 300 high-redshift candidates at $z \sim 6-8$, including the brightest known at $z \sim 6$ (Salmon et al. 2017). These galaxies are lensed as brightly as F160W $H \sim 23$, enabling detailed studies of galaxy properties in the first billion years. Follow-up study is beginning to match some of these candidates as multiple images (Acebron et al. 2018) and deliver spectroscopic confirmations (Cibirka et al. 2018). We are following up RELICS high-redshift candidates with ground-based telescopes and instruments, including Keck/ MOSFIRE, VLT/MUSE, Gemini/GMOS, Gemini-S/Flamingos-2, ALMA, and NOEMA/PdBI. 
Table 15

HST Source Catalog: Detection and Shape Measurement

\begin{tabular}{|c|c|c|c|c|c|c|c|c|c|c|c|}
\hline ID & $\begin{array}{c}\alpha_{\mathrm{J} 2000} \\
(\mathrm{deg})\end{array}$ & $\begin{array}{l}\delta_{\mathrm{J} 2000} \\
(\mathrm{deg})\end{array}$ & $\begin{array}{c}x \\
\text { (pixels) }\end{array}$ & $\begin{array}{c}y \\
\text { (pixels) }\end{array}$ & $\begin{array}{l}\text { FWHM } \\
(\operatorname{arcsec})\end{array}$ & $\begin{array}{c}\text { Area } \\
\text { (pixels) }\end{array}$ & Stellarity & $\begin{array}{l}\text { Ellipticity } \\
(1-b / a)\end{array}$ & $\begin{array}{c}\theta \\
(\mathrm{deg})\end{array}$ & $N_{f, 5 \sigma}$ & $N_{f, \text { obs }}$ \\
\hline 1 & 130.7320691 & 36.3412393 & 3212.886 & 1118.780 & 0.356 & 13 & 0.35 & 0.26 & 11.0 & 1 & 7 \\
\hline 2 & 130.7567476 & 36.3856742 & 2020.442 & 3784.849 & 0.320 & 474 & 0.03 & 0.09 & -42.8 & 7 & 7 \\
\hline 3 & 130.7567488 & 36.3851756 & 2020.382 & 3754.932 & 0.754 & 125 & 0.00 & 0.33 & 58.4 & 7 & 7 \\
\hline 4 & 130.7545731 & 36.3848770 & 2125.475 & 3737.004 & 0.173 & 42 & 0.98 & 0.06 & -10.0 & 5 & 7 \\
\hline 5 & 130.7535624 & 36.3842826 & 2174.292 & 3701.339 & 0.378 & 59 & 0.01 & 0.21 & 80.2 & 7 & 7 \\
\hline
\end{tabular}

Note. Complete RELICS HST catalogs are available on MAST, including all parameters described in Table 14. This sample of the content is from the A697 IRdetection catalog.

Table 16

HST Source Catalog: Photometry in Each Filter

\begin{tabular}{lccc}
\hline \hline $\begin{array}{l}\text { Magnitude } \\
(\mathrm{AB})\end{array}$ & $\begin{array}{c}\text { Flux } \\
\left(e^{-} \mathrm{s}^{-1}\right)\end{array}$ & $\begin{array}{c}\text { Flux } \\
(\mathrm{nJy})\end{array}$ & $\mathrm{S} / \mathrm{N}$ \\
\hline $28.4629 \pm 0.4579$ & $0.0760 \pm 0.0399$ & $14.9573 \pm 7.8473$ & 1.9100 \\
$24.2190 \pm 0.0567$ & $3.7868 \pm 0.2031$ & $745.4248 \pm 39.9764$ & 18.6500 \\
$25.5322 \pm 0.1058$ & $1.1298 \pm 0.1156$ & $222.3921 \pm 22.7527$ & 9.7700 \\
$99.0000 \pm 28.6848$ & $-0.1387 \pm 0.0619$ & $-27.3093 \pm 12.1918$ & -2.2400 \\
$25.6583 \pm 0.0796$ & $1.0059 \pm 0.0765$ & $198.0041 \pm 15.0577$ & 13.1500 \\
\hline
\end{tabular}

Note. Continuation of sample provided in Table 15. Parameters are described in Table 14. The photometry given here is from the F435W filter. In the full catalog, all filters are provided, followed by the brightest magnitude in any filter for reference.

RELICS also delivered SPT0615-JD, the most distant lensed arc known (Salmon et al. 2018). At $z \sim 10$ and spanning a full $2 ! " 5$ on the sky, SPT0615-JD provides by far the most detailed view we have of any galaxy in the first 500 million years. (The two known $z \sim 11$ galaxies are not spatially resolved, despite lensing magnification in the case of MACS0647-JD from Coe et al. 2013 and the relatively high intrinsic luminosity of GNz11 from Oesch et al. 2016.) ALMA observations have been awarded (PI Tamura) to search for the [O III] $88 \mu \mathrm{m}$ line in the $z \sim 10$ lensed arc, continuing the success of that research group at $z \sim 7-9$ as noted in Section 2.1 (Inoue et al. 2016; Hashimoto et al. 2018; Tamura et al. 2019). An [O III] detection would yield the highest spectroscopic redshift confirmation to date, along with the earliest detection of heavy elements (oxygen).

RELICS Spitzer (SRELICS) imaging was crucial in distinguishing between bonafide $z \sim 10$ candidates and $z \sim 2$ interlopers. Salmon et al. (2018) actually identified three $z \sim 10$ candidates based on our HST imaging, but two turned out to be $z \sim 2$ interlopers based on the Spitzer photometry. Red $z \sim 2$ galaxies are significantly brighter at 3-5 $\mu \mathrm{m}$ than bluer $z \sim 10$ galaxies. RELICS Spitzer imaging will also enable us to measure stellar masses for our $>300$ candidates at $z \sim 6-8$.

Improved constraints on the $z \sim 9$ luminosity function from RELICS will require adding simulating lensed galaxies to our images to quantify our detection efficiency as a function of magnitude, position, and redshift (e.g., Livermore et al. 2017; Carrasco et al. 2018). But based on our current lack of any strong $z \sim 9$ candidates and a single $z \sim 10$ candidate (Salmon et al. 2017, 2018), our yields appear lower than expected at these redshifts, which may support the accelerated evolution scenario. Alternatively, more detailed study may shed light on why we have missed galaxies at these redshifts in our searches and photometric redshift analyses.

At lower redshifts, RELICS is studying compact, lowmetallicity dwarf galaxies that are excellent analogs to highredshift galaxies but can be studied in greater detail. Analysis of one RELICS $z=1.645$ galaxy shows that low-metallicity stars are driving $\mathrm{C} \mathrm{III]} \mathrm{emission} \mathrm{with} \mathrm{the} \mathrm{strongest} \mathrm{rest-frame}$ EW ( $\sim 20 \AA)$ yet observed at these redshifts, suggesting a more intense radiation field than assumed by most population synthesis models (Mainali et al. 2019).

\subsection{Lens Modeling}

Strong-lens models of RELICS clusters are primarily used to estimate magnifications of our lensed galaxies and to correct the surveyed volume for lensing magnification (Section 2.2). We use three lens modeling methods, all of which assume that the observed cluster light traces some component of the cluster mass distribution: Lenstool (Kneib et al. 1996; Jullo \& Kneib 2009; Johnson et al. 2014); Zitrin LTM, or lighttraces-mass (Zitrin et al. 2009, 2015b); and GLAFIC (Oguri 2010). The use of multiple methods on an individual cluster yields a more accurate estimate of systematic uncertainties than using one method alone, as shown in analyses of Frontier Fields clusters (Coe et al. 2015; Acebron et al. 2017; Livermore et al. 2017; Remolina González et al. 2018).

RELICS has published strong-lens modeling analyses of 14 clusters to date (Acebron et al. 2018, 2019; Cerny et al. 2018; Cibirka et al. 2018; Paterno-Mahler et al. 2018; Mahler et al. 2019). These models are based on the multiband HST imaging and spectroscopic redshifts of strongly lensed galaxies obtained by our group and others. Most of these analyses have revealed lensing strengths on par with Frontier Fields clusters, quantified in terms of cumulative area with magnification greater than some threshold. Strong-lens models are currently available on MAST for 28/41 RELICS clusters, with the rest to be delivered in time for the JWST GO call for proposals. Our data products include maps of cluster mass, as well as lensing deflection, shear, and magnification.

Paterno-Mahler et al. (2018) used all three methods (primarily Lenstool) to model SPT0615-57 and study the $z \sim 10$ candidate discovered by Salmon et al. (2018). They delivered magnification estimates and explained the current lack of observed counterimages. Acebron et al. (2019) compared magnification estimates from two methods (Zitrin LTM and Lenstool) for the many (24) $z \sim 6-7$ candidates lensed by CL0152-13. Acebron et al. (2018) modeled two clusters with the Zitrin LTM method, including MACS0308 +26 , which lenses one of the brightest $z \sim 6$ candidates known. This paper identified two multiple images of that 
Table 17

HST Source Catalog: Photometric Redshifts

\begin{tabular}{lccccccccc}
\hline \hline$z_{\text {BPZ }}$ & $z_{\min }$ & $z_{\max }$ & $t_{\mathrm{BPZ}}$ & ODDS & $\chi^{2}$ & $\chi_{\bmod }^{2}$ & $M_{0}$ & $z_{\mathrm{ML}}$ \\
\hline 2.741 & 0.225 & 3.470 & 9.5 & 0.148 & 0.940 & 1.334 & 28.102 & 2.730 \\
0.222 & 0.145 & 0.255 & 4.1 & 0.850 & 0.933 & 0.158 & 22.015 & 0.190 \\
1.033 & 0.631 & 1.115 & 9.6 & 0.481 & 0.163 & 0.212 & 25.013 & 1.040 \\
4.501 & 4.315 & 4.698 & 8.2 & 0.974 & 1.292 & 1.367 & 25.835 & 4.520 \\
0.778 & 0.461 & 0.961 & 10.3 & 0.473 & 0.112 & 0.770 & 25.346 & 0.780 \\
\hline
\end{tabular}

Note. Continuation of sample provided in Table 15. Parameters are described in Table 14.

Table 18

RELICS Supernovae and HST Follow-up Imaging

\begin{tabular}{llllll}
\hline \hline Cluster & Supernova $^{\text {a }}$ & Abbreviation $^{\text {b }}$ & R.A. (J2000) & Decl. (J2000) & Notes \\
\hline rxc0949+17 $^{\text {rxc0949+17 }}$ & Eleanor $^{\mathrm{c}}$ & RLC11Ele & $09: 49: 47.97$ & $+17: 07: 24.9$ & cluster member \\
rxc0949+17 & Alexander $^{\mathrm{c}}$ & RLC11Ale & $09: 49: 48.07$ & $+17: 07: 24.0$ & cluster member \\
rxc0142+44 & Antikythera & RLC15Ant & $09: 49: 48.01$ & $+17: 07: 23.0$ & cluster member \\
abell1763 & Makapansgat & RLC16Mak & $01: 43: 16.326$ & $+44: 33: 50.65$ & parallel field \\
macs0025-12 & Nebra & RLC16Neb & $13: 35: 15.13$ & $+41: 00: 15.8$ & lensed \\
macs0257-23 & Quipu & RLC16Qui & $00: 25: 31.977$ & $-12: 23: 31.80$ & cluster member \\
plckg171-40 & Cheomseongdae & RLC16Che & $02: 57: 07.795$ & $-23: 27: 11.69$ & lensed or cluster member \\
clj0152-13 & Kukulkan & RLC16Kuk & $03: 12: 59.148$ & $+08: 22: 43.60$ & cluster member \\
rxc0600-20 & Nimrud & RLC16Nim & $01: 52: 40.352$ & $-13: 57: 44.81$ & lensed \\
smacs0723-73 & William & RLC17Wil & $06: 00: 12.227$ & $-20: 07: 23.91$ & cluster member \\
& Yupana & RLC17Yup & $07: 23: 28.40$ & $-73: 27: 03.6$ & lensed or cluster member \\
\hline
\end{tabular}

Notes.

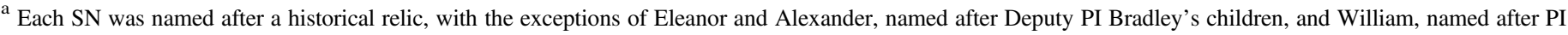
Coe's newborn son.

b Abbreviations include the last two digits of the year of appearance.

${ }^{c}$ Discovered in pre-RELICS imaging based on difference comparison with RELICS imaging.

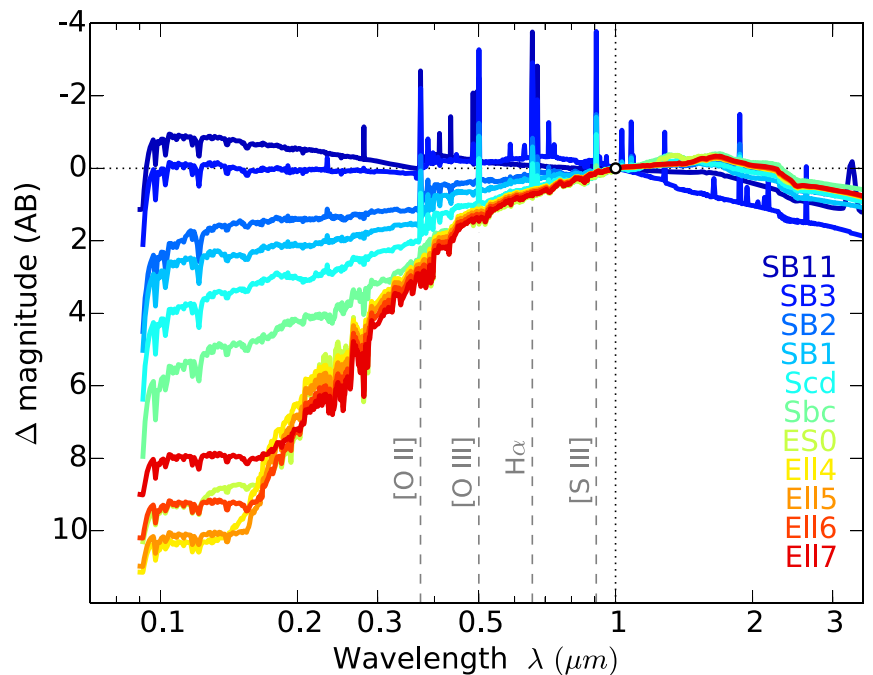

Figure 5. The $11 \mathrm{BPZ}$ template SEDs used in this work, consisting of four elliptical galaxies (Ell), one lenticular (ES0), and four starbursts (SB). These templates are based on PEGASE (Fioc \& Rocca-Volmerange 1997) but recalibrated based on observed photometry and spectroscopic redshifts from FIREWORKS (Wuyts et al. 2008). The starbursts and Scd spiral contain emission lines, four of which are labeled in gray. All spectra are normalized to the same magnitude at $1 \mu \mathrm{m}$.

galaxy lensed to $J \sim 23.2$ and 24.6 AB. Mahler et al. (2019) used spectroscopic redshifts of many arcs from VLT/MUSE (Jauzac et al. 2019) to produce a detailed Lenstool model of MACS0417-11, confirming that it is a strong lens, despite

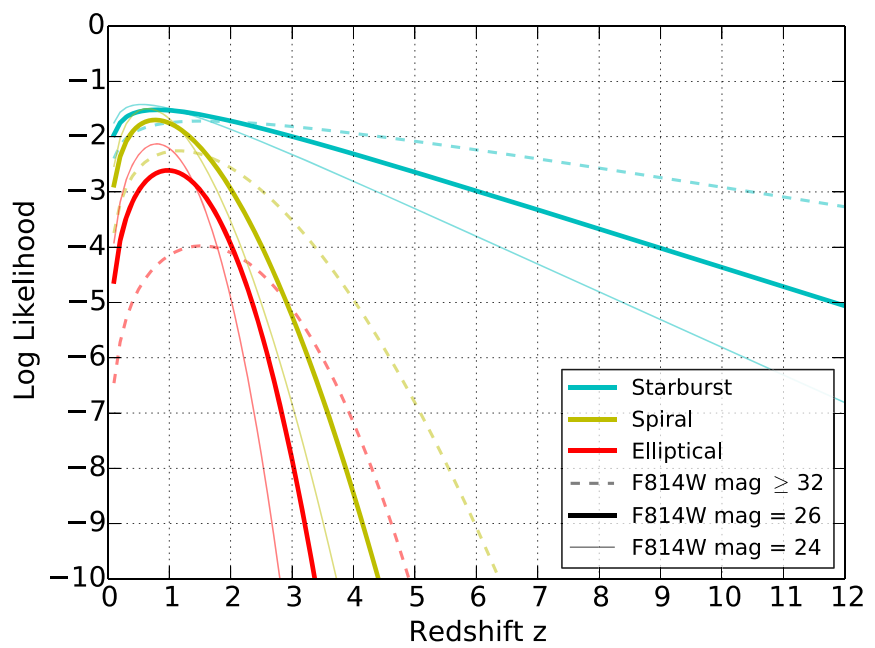

Figure 6. For BPZ, we use the Bayesian redshift prior derived from the HDFN (Benítez 2000). Here we show priors for galaxies of different spectral types and with $\mathrm{F} 814 \mathrm{~W}$ AB magnitudes of 24, 26, and $\geqslant 32$, including nondetections.

curiously yielding no $z \sim 6-8$ candidates (Salmon et al. 2017). Cibirka et al. (2018) modeled four clusters using the LTM method and presented one lensed galaxy showing strong $\operatorname{Ly} \alpha$ emission with a spectroscopic redshift $z=5.800$. Cerny et al. (2018) modeled five clusters using Lenstool and presented detailed estimates of the statistical and systematic uncertainties. They present new spectroscopic redshifts and a new method to mitigate modeling uncertainties due to photometric redshifts. 
Wide-field imaging for weak-lensing analyses from Subaru and Magellan is in hand for many RELICS clusters, as are X-ray data from Chandra and XMM-Newton, along with Planck SZ mass measurements. Combining these with stronglensing mass measurements from HST will improve both precision and accuracy on the overall mass calibration of massive clusters (Section 2.3) and contribute to constraints on the dark matter particle cross section (Section 2.4).

\subsection{Supernovae}

The RELICS observing strategy (Section 2.5) yielded 11 $\mathrm{SNe}$, summarized here for the first time in Table 18. Most of these are cluster members, as expected. The first three $\mathrm{SNe}$, all found in the RXC 0949+17 cluster, were announced in Rodney et al. (2015a). Three of the other RELICS SNe are lensed, and we obtained follow-up HST imaging of them, using 20 orbits allocated to RELICS for this purpose (Table 7). The most distant, dubbed "Nebra," is a $z \sim 2$ candidate Type Ia lensed by A1763 (Rodney et al. 2016a). If the redshift and type were confirmed, Nebra would be among the most distant SNe Ia known. The current record holder at $z=2.22$ is also lensed (Rubin et al. 2018).

\section{Summary}

With RELICS observations complete, the 34 most massive Planck clusters $\left(M_{500}>8.8 \times 10^{14} M_{\odot}\right)$ now all have $H S T$ optical and near-infrared imaging, as well as Spitzer infrared imaging. Based on this imaging, we have discovered over 300 $z \sim 6-10$ candidates, including the brightest galaxies known at $z \sim 6$ (Salmon et al. 2017) and the most distant spatially resolved lensed arc known, SPT0615-JD at $z \sim 10$ (Salmon et al. 2018). These are among the best and brightest targets for detailed follow-up study from the first billion years after the big bang. Follow-up observations of RELICS fields are currently being carried out with facilities including Keck, VLT, Subaru, Magellan, MMT, GMRT, and ALMA.

At lower redshifts, RELICS is studying compact, lowmetallicity dwarf galaxies that are excellent analogs to highredshift galaxies but can be studied in greater detail. We have also discovered $11 \mathrm{SNe}$ (Table 18).

To date, we have published strong-lens modeling analyses of 14 RELICS clusters (Acebron et al. 2018, 2019; Cerny et al. 2018; Cibirka et al. 2018; Paterno-Mahler et al. 2018; Mahler et al. 2019). Many of the clusters modeled so far have proven to be comparable to the strongest lenses known. By combining our strong-lensing analyses of the cluster cores with weaklensing analyses from ground-based imaging covering the full clusters out to their virial radii, we can derive robust mass profiles for these clusters. Our cluster mass measurements will help inform SZ mass scaling relations.

RELICS has proven once again that cluster lensing delivers distant galaxies more efficiently than blank-field observations. The efficiency gains are greatest for discoveries of relatively bright galaxies. We expect these gains to be even greater at higher redshifts. Our lens modeling analyses of RELICS clusters will identify which cluster lenses are truly among the best to use going forward to efficiently search for the first galaxies.

RELICS HST reduced images, catalogs, and lens models are available via MAST at doi:10.17909/T9SP45. RELICS Spitzer reduced images are available via IRSA.
We thank Lindsey Bleem for providing Magellan Megacam LDSS3 images of SPT0254-58 and AS295 prior to RELICS to inform our HST observations of these clusters. We thank Florian Pacaud and Matthias Klein for discussions regarding our AS295 HST pointings. We thank Dale Kocevski for an image of RXC $0142+44$ obtained with the University of Hawaii $2.2 \mathrm{~m}$ telescope. And we thank Stella Seitz et al. for sharing their HST observations of PLCK G287+32 obtained during the same cycle.

We thank the STScI and SSC directors and time allocation committees for enabling these large observing programs. We are grateful to our HST program coordinator William Januszewski for implementing the RELICS HST observations. We thank Jennifer Mack for expert mentoring of our HST image reduction gurus RA and SO. And we thank Gabriel Brammer for providing an updated WFC3/IR hot pixel mask derived from science observations from GO 14114.

We are grateful to the University of Arizona for hosting our team meeting.

The RELICS Hubble Treasury Program (GO 14096) consists of observations obtained by the NASA/ESA Hubble Space Telescope (HST). HST is operated by the Association of Universities for Research in Astronomy, Inc. (AURA), under NASA contract NAS5-26555.

Data from the NASA/ESA $H S T$ presented in this paper were obtained from the Mikulski Archive for Space Telescopes (MAST), operated by the Space Telescope Science Institute (STScI). STScI is operated by the Association of Universities for Research in Astronomy, Inc. (AURA), under NASA contract NAS 5-26555. The HST Advanced Camera for Surveys (ACS) was developed under NASA contract NAS 5-32864.

Spitzer Space Telescope data presented in this paper were obtained from the NASA/IPAC Infrared Science Archive (IRSA), operated by the Jet Propulsion Laboratory, California Institute of Technology. Spitzer and IRSA are operated by the Jet Propulsion Laboratory, California Institute of Technology, under contract with NASA.

We gratefully acknowledge support from JPL for the Spitzer analysis. M.B. and V.S. also acknowledge support by NASA through ADAP grant 80NSSC18K0945, NASA/HST through HST-GO-14096, HST-GO-13666, and two awards issued by Spitzer/JPL/Caltech associated with the SRELICS_DEEP and SRELICS programs.

Part of this work by W.D. was performed under the auspices of the U.S. DOE by LLNL under contract DE-AC5207NA27344. K.U. acknowledges support from the Ministry of Science and Technology of Taiwan (grant MOST 106-2628M-001-003-MY3) and from Academia Sinica (grant AS-IA107-M01). O.G. is supported by an NSF Astronomy and Astrophysics Fellowship under award AST-1602595. S.A.R. was supported by NASA grant HST-GO-14208 from STScI, which is operated by Associated Universities for Research in Astronomy, Inc. (AURA), under NASA contract NAS 5-26555. A.M. acknowledges the financial support of the Brazilian funding agency FAPESP (Post-doc fellowshipprocess No. 2014/11806-9). J.H. was supported by a VILLUM FONDEN Investigator grant (project No. 16599).

Finally, we thank our referee for detailed comments that helped us improve the manuscript.

Facilities: HST (WFC3, ACS); Spitzer (IRAC); Planck; MAST; IRSA. 


\section{ORCID iDs}

Dan Coe (i) https://orcid.org/0000-0001-7410-7669 Brett Salmon (iD https://orcid.org/0000-0002-7453-7279 Maruša Bradač (i) https://orcid.org/0000-0001-5984-0395 Larry D. Bradley (iD https://orcid.org/0000-0002-7908-9284 Keren Sharon (iD https://orcid.org/0000-0002-7559-0864 Adi Zitrin (i) https://orcid.org/0000-0002-0350-4488 Ana Acebron (iD https://orcid.org/0000-0003-3108-9039 Nathália Cibirka (iD https://orcid.org/0000-0002-2356-4680 Victoria Strait (iD https://orcid.org/0000-0002-6338-7295 Rachel Paterno-Mahler (i) https://orcid.org/0000-0003-3653-3741 Guillaume Mahler (i) https://orcid.org/0000-0003-3266-2001 Kuang-Han Huang (iD https://orcid.org/0000-0001-7826-6448 Ramesh Mainali (i) https://orcid.org/0000-0003-0094-6827 Pascal A. Oesch (D) https://orcid.org/0000-0001-5851-6649 Michele Trenti (iD https://orcid.org/0000-0001-9391-305X Daniela Carrasco (iD https://orcid.org/0000-0002-3772-0330 William A. Dawson (iD https://orcid.org/0000-0003-0248-6123 Steven A. Rodney (iD https://orcid.org/0000-0003-1947-687X Keiichi Umetsu (1) https://orcid.org/0000-0002-7196-4822 Benedetta Vulcani (i) https://orcid.org/0000-0003-0980-1499 Or Graur (iD https://orcid.org/0000-0002-4391-6137 Saurabh W. Jha (i) https://orcid.org/0000-0001-8738-6011 Melissa L. Graham (i) https://orcid.org/0000-0002-9154-3136 Jens Hjorth (iD https://orcid.org/0000-0002-4571-2306 Jonatan Selsing (iD https://orcid.org/0000-0001-9058-3892 Lise Christensen (i) https://orcid.org/0000-0001-8415-7547 Shotaro Kikuchihara (iD https://orcid.org/0000-0003-2449-6314 Masami Ouchi (iD https://orcid.org/0000-0002-1049-6658 Masamune Oguri (iD https://orcid.org/0000-0003-3484-399X Felipe Andrade-Santos (iD https://orcid.org/0000-0002-8144-9285 Austin T. Hoag (iD https://orcid.org/0000-0001-8989-2567 Traci L. Johnson (iD https://orcid.org/0000-0002-8829-5303 Rachael Livermore (i) https://orcid.org/0000-0003-4456-1566 Daniel Lam (i) https://orcid.org/0000-0002-6536-5575 Sune Toft (ib https://orcid.org/0000-0003-3631-7176

\section{References}

Abell, G. O. 1958, ApJS, 3, 211

Abell, G. O., Corwin, H. G., Jr., \& Olowin, R. P. 1989, ApJS, 70, 1 Acebron, A., Alon, M., Zitrin, A., et al. 2019, ApJ, 874, 132 Acebron, A., Cibirka, N., Zitrin, A., et al. 2018, ApJ, 858, 42 Acebron, A., Jullo, E., Limousin, M., et al. 2017, MNRAS, 470, 1809 Albert, J. G., Sifón, C., Stroe, A., et al. 2017, A\&A, 607, A4 Applegate, D. E., von der Linden, A., Kelly, P. L., et al. 2014, MNRAS, 439, 48 Atek, H., Richard, J., Kneib, J.-P., \& Schaerer, D. 2018, MNRAS, 479, 5184 Battaglia, N., Leauthaud, A., Miyatake, H., et al. 2016, JCAP, 2016, 013 Benítez, N. 2000, ApJ, 536, 571

Benítez, N., Dupke, R., Moles, M., et al. 2014, arXiv:1403.5237 Bertin, E., \& Arnouts, S. 1996, A\&AS, 117, 393

Bleem, L. E., Stalder, B., de Haan, T., et al. 2015, ApJS, 216, 27 Bosch-Ramon, V. 2018, A\&A, 617, L3

Bouwens, R. J., Bradley, L., Zitrin, A., et al. 2014, ApJ, 795, 126 Bouwens, R. J., Illingworth, G. D., Oesch, P. A., et al. 2012, ApJ, 754, 83 Bouwens, R. J., Illingworth, G. D., Oesch, P. A., et al. 2015, ApJ, 803, 34 Bradač, M., Allen, S. W., Treu, T., et al. 2008, ApJ, 687, 959 Bradač, M., Clowe, D., Gonzalez, A. H., et al. 2006, ApJ, 652, 937 Bradač, M., Ryan, R., Casertano, S., et al. 2014, ApJ, 785, 108 Bradač, M., Vanzella, E., Hall, N., et al. 2012, ApJL, 755, L7 Bradley, L. D., Bouwens, R. J., Ford, H. C., et al. 2008, ApJ, 678, 647 Bradley, L. D., Trenti, M., Oesch, P. A., et al. 2012, ApJ, 760, 108 Bradley, L. D., Zitrin, A., Coe, D., et al. 2014, ApJ, 792, 76 Brammer, G. B., Pirzkal, N., McCullough, P., \& MacKenty, J. W. 2014, WFC3 Instrument Science Rep. 2014-03, Tech. Rep., STScI

Brammer, G. B., van Dokkum, P. G., \& Coppi, P. 2008, ApJ, 686, 1503 Brauher, J. R., Dale, D. A., \& Helou, G. 2008, ApJS, 178, 280
Broadhurst, T. J., Taylor, A. N., \& Peacock, J. A. 1995, ApJ, 438, 49 Calvi, V., Trenti, M., Stiavelli, M., et al. 2016, ApJ, 817, 120

Capak, P. L., Carilli, C., Jones, G., et al. 2015, Natur, 522, 455 Carniani, S., Maiolino, R., Amorin, R., et al. 2018, MNRAS, 478, 1170 Carniani, S., Maiolino, R., Pallottini, A., et al. 2017, A\&A, 605, A42 Carrasco, D., Trenti, M., Mutch, S., \& Oesch, P. A. 2018, PASA, 35, e022 Cerny, C., Sharon, K., Andrade-Santos, F., et al. 2018, ApJ, 859, 159 Chan, B. M. Y., Broadhurst, T., Lim, J., et al. 2017, ApJ, 835, 44 Chen, W., Kelly, P. L., Diego, J. M., et al. 2019, ApJ, 881, 8 Cibirka, N., Acebron, A., Zitrin, A., et al. 2018, ApJ, 863, 145 Clowe, D., Bradač, M., Gonzalez, A. H., et al. 2006, ApJL, 648, L109 Coe, D. 2010, arXiv:1005.0411

Coe, D. 2018, SciAm, 319, 40

Coe, D., Benítez, N., Sánchez, S. F., et al. 2006, AJ, 132, 926 Coe, D., Bradley, L., \& Zitrin, A. 2015, ApJ, 800, 84

Coe, D., Umetsu, K., Zitrin, A., et al. 2012, ApJ, 757, 22 Coe, D., Zitrin, A., Carrasco, M., et al. 2013, ApJ, 762, 32 Czakon, N. G., Sayers, J., Mantz, A., et al. 2015, ApJ, 806, 18 Dawson, W. A., Wittman, D., Jee, M. J., et al. 2012, ApJL, 747, L42 Dayal, P., \& Ferrara, A. 2018, PhR, 780, 1

De Looze, I., Cormier, D., Lebouteiller, V., et al. 2014, A\&A, 568, A62 Du, X., Shapley, A. E., Martin, C. L., \& Coil, A. L. 2017, ApJ, 838, 63 Ebeling, H., Barrett, E., Donovan, D., et al. 2007, ApJL, 661, L33 Ebeling, H., Edge, A. C., Allen, S. W., et al. 2000a, MNRAS, 318, 333 Ebeling, H., Edge, A. C., Bohringer, H., et al. 1998, MNRAS, 301, 881 Ebeling, H., Edge, A. C., Burgett, W. S., et al. 2013, MNRAS, 432, 62 Ebeling, H., Edge, A. C., \& Henry, J. P. 2001, ApJ, 553, 668 Ebeling, H., Edge, A. C., Mantz, A., et al. 2010, MNRAS, 407, 83 Ebeling, H., Jones, L. R., Perlman, E., et al. 2000b, ApJ, 534, 133 Erb, D. K., Pettini, M., Shapley, A. E., et al. 2010, ApJ, 719, 1168 Finkelstein, S. L. 2016, PASA, 33, e037

Finkelstein, S. L., D’Aloisio, A., Paardekooper, J.-P., et al. 2019, ApJ, 879, 36

Fioc, M., \& Rocca-Volmerange, B. 1997, A\&A, 326, 950

Franx, M., Illingworth, G. D., Kelson, D. D., van Dokkum, P. G., \& Tran, K.-V. 1997, ApJL, 486, L75

Gioia, I. M., Maccacaro, T., Schild, R. E., et al. 1990, ApJS, 72, 567

Gonzaga, S., Hack, W., Fruchter, A., \& Mack, J. 2012, The DrizzlePac Handbook (Baltimore, MD: STScI)

Grandis, S., Mohr, J. J., Dietrich, J. P., et al. 2019, MNRAS, 488, 2041

Graur, O., Rodney, S. A., Maoz, D., et al. 2014, ApJ, 783, 28

Grillo, C., Rosati, P., Suyu, S. H., et al. 2018, ApJ, 860, 94

Grogin, N. A., Kocevski, D. D., Faber, S. M., et al. 2011, ApJS, 197, 35

Gruen, D., Seitz, S., Becker, M. R., Friedrich, O., \& Mana, A. 2015, MNRAS, 449,4264

Hamilton-Morris, V., Smith, G. P., Edge, A. C., et al. 2012, ApJL, 748, L23 Harikane, Y., Ouchi, M., Shibuya, T., et al. 2018, ApJ, 859, 84

Harvey, D., Massey, R., Kitching, T., Taylor, A., \& Tittley, E. 2015, Sci, 347,1462

Hashimoto, T., Inoue, A. K., Mawatari, K., et al. 2019, PASJ, 71, 71

Hashimoto, T., Laporte, N., Mawatari, K., et al. 2018, Natur, 557, 392 Hasselfield, M., Hilton, M., Marriage, T. A., et al. 2013, JCAP, 7, 008 Hildebrandt, H., Arnouts, S., Capak, P., et al. 2010, A\&A, 523, A31 Hoag, A., Bradač, M., Huang, K.-H., et al. 2019, ApJ, 878, 12

Hoag, A., Bradač, M., Trenti, M., et al. 2017, NatAs, 1, 0091 Hoekstra, H., Herbonnet, R., Muzzin, A., et al. 2015, MNRAS, 449, 685 Hsu, L.-Y., Ebeling, H., \& Richard, J. 2013, MNRAS, 429, 833 Huang, K.-H., Bradač, M., Lemaux, B. C., et al. 2016, ApJ, 817, 11 Infante, L., Zheng, W., Laporte, N., et al. 2015, ApJ, 815, 18 Inoue, A. K., Shimizu, I., Tamura, Y., et al. 2014, ApJL, 780, L18 Inoue, A. K., Tamura, Y., Matsuo, H., et al. 2016, Sci, 352, 1559 Ishigaki, M., Kawamata, R., Ouchi, M., et al. 2018, ApJ, 854, 73 Izotov, Y. I., Worseck, G., Schaerer, D., et al. 2018, MNRAS, 478, 4851 Jauzac, M., Mahler, G., Edge, A. C., et al. 2019, MNRAS, 483, 3082 Johnson, T. L., Rigby, J. R., Sharon, K., et al. 2017, ApJL, 843, L21 Johnson, T. L., \& Sharon, K. 2016, ApJ, 832, 82

Johnson, T. L., Sharon, K., Bayliss, M. B., et al. 2014, ApJ, 797, 48 Jullo, E., \& Kneib, J. 2009, MNRAS, 395, 1319

Kaurov, A. A., Dai, L., Venumadhav, T., Miralda-Escudé, J., \& Frye, B. 2019, ApJ, 880, 58

Kausch, W., Schindler, S., Erben, T., Wambsganss, J., \& Schwope, A. 2010, A\&A, 513, A8

Kawamata, R., Ishigaki, M., Shimasaku, K., et al. 2018, ApJ, 855, 4 Kelly, P. L., Diego, J. M., Rodney, S., et al. 2018, NatAs, 2, 334 Kelly, P. L., Rodney, S. A., Treu, T., et al. 2015, Sci, 347, 1123 Kelly, P. L., Rodney, S. A., \& Treu, T. 2016, ApJL, 819, L8 
Kneib, J.-P., Ellis, R. S., Smail, I., Couch, W. J., \& Sharples, R. M. 1996, ApJ, 471,643

Kneib, J.-P., \& Natarajan, P. 2011, A\&ARv, 19, 47

Kocevski, D. D., Ebeling, H., Mullis, C. R., \& Tully, R. B. 2007, ApJ, 662,224

Koekemoer, A. M., Faber, S. M., Ferguson, H. C., et al. 2011, ApJS, 197, 36

Koekemoer, A. M., Fruchter, A. S., Hook, R. N., \& Hack, W. 2002 MultiDrizzle: An Integrated Pyraf Script for Registering, Cleaning and Combining Images, in The 2002 HST Calibration Workshop, ed. S. Arribas, A. Koekemoer, \& B. Whitmore (Baltimore, MD: STSci), 337

Laporte, N., Ellis, R. S., Boone, F., et al. 2017a, ApJL, 837, L21

Laporte, N., Nakajima, K., Ellis, R. S., et al. 2017b, ApJ, 851, 40

Le Fèvre, O., Lemaux, B. C., Nakajima, K., et al. 2019, A\&A, 625, 51

Leitherer, C., Hernandez, S., Lee, J. C., \& Oey, M. S. 2016, ApJ, 823, 64

Livermore, R. C., Finkelstein, S. L., \& Lotz, J. M. 2017, ApJ, 835, 113

Lotz, J. M., Koekemoer, A., Coe, D., et al. 2017, ApJ, 837, 97

Madau, P. 2017, ApJ, 851, 50

Mahler, G., Sharon, K., Fox, C., et al. 2019, ApJ, 873, 96

Mainali, R., Kollmeier, J. A., Stark, D. P., et al. 2017, ApJL, 836, L14

Mainali, R., Stark, D. P., Tang, M., et al. 2019, MNRAS, submitted

Mainali, R., Zitrin, A., Stark, D. P., et al. 2018, MNRAS, 479, 1180

Makovoz, D., \& Khan, I. 2005, in ASP Conf. Ser. 347, Astronomical Data Analysis Software and Systems XIV, ed. P. Shopbell, M. Britton, \& R. Ebert (San Francisco, CA: ASP), 81

Malhotra, S., Helou, G., Stacey, G., et al. 1997, ApJL, 491, L27

Mann, A. W., \& Ebeling, H. 2012, MNRAS, 420, 2120

Mantz, A., Allen, S. W., Ebeling, H., Rapetti, D., \& Drlica-Wagner, A. 2010, MNRAS, 406, 1773

Maraston, C. 2005, MNRAS, 362, 799

Marrone, D. P., Spilker, J. S., Hayward, C. C., et al. 2018, Natur, 553, 51

Mason, C. A., Fontana, A., Treu, T., et al. 2019, MNRAS, 485, 3947

Mason, C. A., Treu, T., Dijkstra, M., et al. 2018, ApJ, 856, 2

McLeod, D. J., McLure, R. J., \& Dunlop, J. S. 2016, MNRAS, 459, 3812

Medezinski, E., Battaglia, N., Umetsu, K., et al. 2018, PASJ, 70, S28

Meneghetti, M., Fedeli, C., Pace, F., Gottlöber, S., \& Yepes, G. 2010, A\&A, 519, A90

Meneghetti, M., Natarajan, P., Coe, D., et al. 2017, MNRAS, 472, 3177

Merlin, E., Fontana, A., Ferguson, H. C., et al. 2015, A\&A, 582, A15

Molino, A., Benítez, N., Ascaso, B., et al. 2017, MNRAS, 470, 95

Morishita, T., Trenti, M., Stiavelli, M., et al. 2018, ApJ, 867, 150

Nagai, D., Vikhlinin, A., \& Kravtsov, A. V. 2007, ApJ, 655, 98

Nakajima, K., Schaerer, D., Le Fèvre, O., et al. 2018, A\&A, 612, A94

Nordin, J., Rubin, D., Richard, J., et al. 2014, MNRAS, 440, 2742

Oesch, P. A., Bouwens, R. J., Illingworth, G. D., et al. 2014, ApJ, 786, 108

Oesch, P. A., Bouwens, R. J., Illingworth, G. D., Labbé, I., \& Stefanon, M. 2018, ApJ, 855, 105

Oesch, P. A., Brammer, G., van Dokkum, P. G., et al. 2016, ApJ, 819, 129

Oesch, P. A., van Dokkum, P. G., Illingworth, G. D., et al. 2015, ApJL, 804, L30

Oguri, M. 2010, PASJ, 62, 1017

Oke, J. B. 1974, ApJS, 27, 21

Oke, J. B., \& Gunn, J. E. 1983, ApJ, 266, 713

Patel, B., McCully, C., Jha, S. W., et al. 2014, ApJ, 786, 9

Paterno-Mahler, R., Sharon, K., Coe, D., et al. 2018, ApJ, 863, 154

Penna-Lima, M., Bartlett, J. G., Rozo, E., et al. 2017, A\&A, 604, A89

Piffaretti, R., Arnaud, M., Pratt, G. W., Pointecouteau, E., \& Melin, J.-B. 2011, A\&A, 534, A109

Pillepich, A., Reiprich, T. H., Porciani, C., Borm, K., \& Merloni, A. 2018, MNRAS, 481, 613

Pirzkal, N., Coe, D., Frye, B. L., et al. 2015, ApJ, 804, 11

Planck Collaboration, Ade, P. A. R., Aghanim, N., et al. 2011, A\&A, 536, A11

Planck Collaboration, Ade, P. A. R., Aghanim, N., et al. 2014, A\&A, 571, A29

Planck Collaboration, Ade, P. A. R., Aghanim, N., et al. 2016a, A\&A, 594, A27

Planck Collaboration, Ade, P. A. R., Aghanim, N., et al. 2016b, A\&A, 594, A13

Planck Collaboration, Ade, P. A. R., Aghanim, N., et al. 2016c, A\&A, 594, A24

Planck Collaboration, Ade, P. A. R., Aghanim, N., et al. 2016d, A\&A, 586, A139

Planck Collaboration, Aghanim, N., \& Akrami, Y. 2018a, arXiv:1807.06209

Planck Collaboration, Aghanim, N., Ashdown, M., et al. 2016e, A\&A, 596, A107

Planck Collaboration, Akrami, Y., Arroja, F., et al. 2018b, arXiv:1807.06205

Postman, M., Coe, D., Benítez, N., et al. 2012, ApJS, 199, 25

Puschnig, J., Hayes, M., Östlin, G., et al. 2017, MNRAS, 469, 3252

Rafelski, M., Teplitz, H. I., Gardner, J. P., et al. 2015, AJ, 150, 31
Randall, S. W., Markevitch, M., Clowe, D., Gonzalez, A. H., \& Bradač, M. 2008, ApJ, 679, 1173

Rasia, E., Meneghetti, M., Martino, R., et al. 2012, NJPh, 14, 055018

Remolina González, J. D., Sharon, K., \& Mahler, G. 2018, ApJ, 863, 60

Repp, A., \& Ebeling, H. 2018, MNRAS, 479, 844

Repp, A., Ebeling, H., \& Richard, J. 2016, MNRAS, 457, 1399

Rigby, J. R., Bayliss, M. B., Gladders, M. D., et al. 2015, ApJL, 814, L6

Rivera-Thorsen, T. E., Dahle, H., Gronke, M., et al. 2017, A\&A, 608, L4

Roberts-Borsani, G. W., Bouwens, R. J., Oesch, P. A., et al. 2016, ApJ, 823,143

Robertson, A., Harvey, D., Massey, R., et al. 2019, MNRAS, 488, 3646

Robertson, B. E., Ellis, R. S., Furlanetto, S. R., \& Dunlop, J. S. 2015, ApJL, 802, L19

Rodney, S., Bradley, L., Coe, D., et al. 2015a, ATel, 8170, 1

Rodney, S., Coe, D., Bradley, L., et al. 2016a, ATel, 9224, 1

Rodney, S. A., Balestra, I., Bradac, M., et al. 2018, NatAs, 2, 324

Rodney, S. A., Patel, B., Scolnic, D., et al. 2015b, ApJ, 811, 70

Rodney, S. A., Riess, A. G., Strolger, L.-G., et al. 2014, AJ, 148, 13

Rodney, S. A., Strolger, L. G., Kelly, P. L., et al. 2016b, ApJ, 820, 50

Rubin, D., Hayden, B., Huang, X., et al. 2018, ApJ, 866, 65

Ryan, R. E., Jr., Gonzalez, A. H., Lemaux, B. C., et al. 2014, ApJL, 786, L4

Salmon, B., Coe, D., Bradley, L., et al. 2017, arXiv:1710.08930

Salmon, B., Coe, D., Bradley, L., et al. 2018, ApJL, 864, L22

Schenker, M. A., Ellis, R. S., Konidaris, N. P., \& Stark, D. P. 2014, ApJ, 795, 20

Schlafly, E. F., \& Finkbeiner, D. P. 2011, ApJ, 737, 103

Schlegel, D. J., Finkbeiner, D. P., \& Davis, M. 1998, ApJ, 500, 525

Schmidt, K. B., Huang, K.-H., Treu, T., et al. 2017, ApJ, 839, 17

Schmidt, K. B., Treu, T., Bradač, M., et al. 2016, ApJ, 818, 38

Senchyna, P., Stark, D. P., Vidal-García, A., et al. 2017, MNRAS, 472, 2608

Sereno, M. 2015, MNRAS, 450, 3665

Sereno, M., \& Ettori, S. 2017, MNRAS, 468, 3322

Shapley, A. E., Steidel, C. C., Strom, A. L., et al. 2016, ApJL, 826, L24

Sharon, K., Gladders, M. D., Rigby, J. R., et al. 2012, ApJ, 746, 161

Smit, R., Bouwens, R. J., Carniani, S., et al. 2018, Natur, 553, 178

Smith, G. P., Mazzotta, P., Okabe, N., et al. 2016, MNRAS, 456, L74

Stacey, G. J., Hailey-Dunsheath, S., Ferkinhoff, C., et al. 2010, ApJ, 724, 957

Stark, D. P. 2016, ARA\&A, 54, 761

Stark, D. P., Ellis, R. S., Charlot, S., et al. 2017, MNRAS, 464, 469

Stark, D. P., Ellis, R. S., Chiu, K., Ouchi, M., \& Bunker, A. 2010, MNRAS, 408, 1628

Stark, D. P., Richard, J., Charlot, S., et al. 2015, MNRAS, 450, 1846

Stark, D. P., Richard, J., Siana, B., et al. 2014, MNRAS, 445, 3200

Strolger, L.-G., Dahlen, T., Rodney, S. A., et al. 2015, ApJ, 813, 93

Sunyaev, R. A., \& Zeldovich, Y. B. 1970, Ap\&SS, 7, 3

Tamura, Y., Mawatari, K., Hashimoto, T., et al. 2019, ApJ, 874, 27

Tilvi, V., Papovich, C., Finkelstein, S. L., et al. 2014, ApJ, 794, 5

Tokunaga, A. T., \& Vacca, W. D. 2005, PASP, 117, 421

Treu, T., Brammer, G., Diego, J. M., et al. 2016, ApJ, 817, 60

Treu, T., Schmidt, K. B., Brammer, G. B., et al. 2015, ApJ, 812, 114

Treu, T., Schmidt, K. B., Trenti, M., Bradley, L. D., \& Stiavelli, M. 2013, ApJL, 775, L29

Umetsu, K., Broadhurst, T., Zitrin, A., Medezinski, E., \& Hsu, L. 2011, ApJ, 729,127

Umetsu, K., Medezinski, E., Nonino, M., et al. 2014, ApJ, 795, 163

Umetsu, K., Zitrin, A., Gruen, D., et al. 2016, ApJ, 821, 116

van Weeren, R. J., Röttgering, H. J. A., Intema, H. T., et al. 2012, A\&A, 546, A124

Vanzella, E., Nonino, M., Cupani, G., et al. 2018, MNRAS, 476, L15

Vega-Ferrero, J., Diego, J. M., Miranda, V., \& Bernstein, G. M. 2018, ApJ, 853, L31

von der Linden, A., Allen, M. T., Applegate, D. E., et al. 2014a, MNRAS, 439, 2 von der Linden, A., Mantz, A., Allen, S. W., et al. 2014b, MNRAS, 443, 1973 Watson, D., Christensen, L., Knudsen, K. K., et al. 2015, Natur, 519, 327

Wen, Z. L., Han, J. L., \& Liu, F. S. 2012, ApJS, 199, 34

Windhorst, R. A., Timmes, F. X., Wyithe, J. S. B., et al. 2018, ApJS, 234, 41

Wittman, D., Golovich, N., \& Dawson, W. A. 2018, ApJ, 869, 104

Wong, K. C., Zabludoff, A. I., Ammons, S. M., et al. 2013, ApJ, 769, 52

Wright, E. L., Eisenhardt, P. R. M., Mainzer, A. K., et al. 2010, AJ, 140, 1868

Wuyts, S., Labbé, I., Schreiber, N. M. F., et al. 2008, ApJ, 682, 985

Zheng, W., Postman, M., Zitrin, A., et al. 2012, Natur, 489, 406

Zitrin, A., Broadhurst, T., Umetsu, K., et al. 2009, MNRAS, 396, 1985

Zitrin, A., Fabris, A., Merten, J., et al. 2015a, ApJ, 801, 44

Zitrin, A., Labbé, I., Belli, S., et al. 2015b, ApJL, 810, L12

Zitrin, A., Seitz, S., Monna, A., et al. 2017, ApJL, 839, L11

Zitrin, A., Zheng, W., Broadhurst, T., et al. 2014, ApJL, 793, L12 